UNIVERSIDADE DE SÃO PAULO
HOSPITAL DE REABILITAÇÃO DE ANOMALIAS CRANIOFACIAIS

BRUNA TOZZETTI ALVES

Achados audiológicos e malformações auriculares no espectro oculoauriculovertebral 

BRUNA TOZZETTI ALVES

\title{
Achados audiológicos e malformações auriculares no espectro oculoauriculovertebral
}

\author{
Dissertação apresentada ao Hospital de \\ Reabilitação de Anomalias Craniofaciais da \\ Universidade de São Paulo para obtenção do título \\ de Mestre em Ciências da Reabilitação. \\ Área de Concentração: Fissuras Orofaciais e \\ Anomalias Relacionadas \\ Orientadora: Profa. Dra. Mariza Ribeiro Feniman
}

\section{BAURU}




\section{UNIVERSIDADE DE SÃO PAULO \\ HOSPITAL DE REABILITAÇÃO DE ANOMALIAS CRANIOFACIAIS}

R. Silvio Marchione, 3-20

Caixa Postal: 1501

17012-900 - Bauru - SP - Brasil

Telefone: (14) 3235-8000

Prof. Dr. Marco Antonio Zago - Reitor da USP

Dra. Maria Aparecida de Andrade Moreira Machado - Superintendente do HRAC /USP

Autorizo, exclusivamente, para fins acadêmicos e científicos, a

reprodução total ou parcial desta Tese.

Bruna Tozzetti Alves

Bauru, de de 2017.

Alves, Bruna Tozzetti

A87a Achados audiológicos e malformações auriculares no espectro oculoauriculovertebral / Bruna Tozzetti Alves. Bauru, 2017.

80p.; il., figs, tabs; $30 \mathrm{~cm}$.

Dissertação de Mestrado - Área de Concentração: Fissuras Orofaciais e Anomalias Relacionadas Hospital de Reabilitação de Anomalias Craniofaciais, Universidade de São Paulo.

Orientador: Mariza Ribeiro Feniman

Descritores: 1. Espectro oculoauriculovertebral 2. Avaliação audiológica 3. Malformações auriculares 4. Ossos temporais 


\section{FOLHA DE APROVAÇÃO}

\section{Bruna Tozzetti Alves}

Dissertação apresentada ao Hospital de Reabilitação de Anomalias Craniofaciais da Universidade de São Paulo para a obtenção do título de Mestre.

Área de Concentração: Fissuras Orofaciais e Anomalias Relacionadas

Aprovado em:

Banca Examinadora

Prof. Dr.

Instituição

Prof. Dr.

Instituição

Profa. Dra. Mariza Ribeiro Feniman

Faculdade de Odontologia de Bauru - FOB-USP

Profa. Dra. Ana Paula Fukushiro

Presidente da Comissão de Pós-Graduação do HRAC-USP

Data de depósito da dissertação junto à SPG: 



\section{DEDICATÓRIA}

Dedico este trabalho aos meus pais Cícero e Zilda, aos meus irmãos Rafael e Leonardo e, ao meu namorado Carlos Henrique. Eu sei que vocês não mediram esforços para que eu estivesse aqui. É por vocês que que acordo todos os dias e me esforço para ser uma pessoa melhor. Obrigada pela confiança! Não há como mensurar o amor que sinto por vocês! 



\section{AGRADECIMENTOS}

"Quando agradecemos, estamos nos desvencilhando de um dos vícios mais perigosos de nosso tempo, a autossuficiência. Como é triste alguém pensar que não precisa de ninguém para viver. Agradecer é estar certo que alguém fez a diferença em sua vida" (Ederson larochevski).

Agradeço em primeiro lugar a Deus, que se mostrou criador, que foi criativo.

Seu fôlego de vida em mim foi sustento e me deu coragem para questionar realidades e propor sempre um novo mundo de possibilidades.

Aos meus pais Cícero e Zilda pelo incentivo e apoio incondicional. Aos meus irmãos Rafael e Leonardo, por sempre estarem ao meu lado, a minha eterna gratidão à vocês, que são a minha razão de viver. Amo vocês!

Ao meu namorado Carlos Henrique pelo companheirismo, amor e paciência nesses últimos meses. À você, todo o meu amor e gratidão!

À todos os meus familiares, principalmente aos meus avôs que desde sempre me mostraram o valor da simplicidade e de um sorriso, em especial, a memória do meu avô Antenor Tozzetti que é a razão por eu estar aqui hoje, na Fonoaudiologia.

À todos os meus amigos garcenses e bauruenses, principalmente as minhas duas amigas/irmãs e meu irmão de coração, Camila Corrêa, Maria Renata José e Rafael Ferreira, por compartilharem comigo a verdadeira amizade em todos os momentos.

À Dra. Melissa Zattoni Antoneli co-orientadora deste trabalho, minha eterna gratidão por todo o ensinamento, paciência, competência partilhada e discussões proporcionadas, principalmente ao despertar o encantamento pelas anomalias craniofaciais. Ao companheirismo, a amizade sincera, as alegrias e desafios do dia a dia, me alegrando e ouvindo sempre. À você toda a minha admiração!

À Profa. Dra. Mariza Ribeiro Feniman orientadora desde trabalho, obrigada por ter me aceito como orientanda nesses dois anos, e pela dedicação para a conclusão do trabalho. 

Ao Dr. Luiz Fernando Manzoni Lourençone, pela ajuda durante a coleta e análise das tomografias de ossos temporais, além de todo o seu conhecimento passado.

À Flávia Maria Ravagnani Neves Cintra, pelas análises estatísticas realizadas durante todo o mestrado.

À toda Equipe Craniomaxilofacial, pelo acolhimento e ensinamentos que vivenciei no período em que participei desta. Vocês me mostraram o verdadeiro significado do trabalho interdisciplinar. Gratidão eterna por poder acompanhar o dia a dia de vocês.

À todas as fonoaudiólogas do Setor de Fonoaudiologia, por enriquecerem valiosamente meu aprendizado, principalmente na prática clínica, em especial às fonoaudiólogas Haline Miguel e Andrea Farah, obrigada pela paciência e ensinamentos para vida!

Aos meus amigos da Pós-graduação do HRAC/USP, em especial à Ana Flávia Rodrigues, Francely Farinha, Gabriela Cavalheiro, Gesiane Cristina e Laiza Vilela pelo companheirismo, amizade e carinho durante toda nossa caminhada.

Às funcionárias da Pós - graduação do HRAC/USP Maria José, Ana Regina e Lucy muito obrigada pela atenção, paciência e colaboração durante esses dois anos de Mestrado.

Ao Hospital de Reabilitação de Anomalias Craniofaciais (HRAC-USP), seus diversos setores, equipes, funcionários e pacientes, pelo espaço, acolhimento e oportunidade de aprendizado.

À Coordenação de Aperfeiçoamento de Pessoal de Nível Superior (CAPES) pela concessão da bolsa de mestrado. 

"Conheça todas as teorias, domine todas as técnicas, mas ao tocar uma alma humana, seja apenas outra alma humana" - Carl Jung 



\section{RESUMO}

O espectro oculoauriculovertebral (EOAV) é um defeito congênito que envolve estruturas derivadas do primeiro e segundo arcos faríngeos e tem como principais características malformação da orelha externa, hipoplasia mandibular, dermóide epibulbar e anomalia de coluna cervical. Orelha externa, média e interna podem ser afetadas nesta condição, e a microtia com atresia do meato acústico externo é o achado mais comum. Perda auditiva condutiva e mista são frequentemente descritas. Objetivos: Verificar a presença de malformações auriculares e a presença de perda auditiva, investigando possível associação entre elas, e verificar a associação entre os achados tomográficos dos ossos temporais com a função auditiva e malformações auriculares em pacientes com diagnóstico de EOAV. Material e Métodos: Foi realizado no Setor de Fonoaudiologia do Hospital de Reabilitação de Anomalias Craniofaciais (HRAC), da Universidade de São Paulo (USP) e envolveu análise retrospectiva de prontuários. A casuística incluiu 72 pacientes com EOAV atualmente acompanhados pela equipe de cirurgia craniomaxilofacial do hospital, que apresentaram em sua documentação clínica dados quanto à presença e ao tipo de malformação auricular, avaliação audiológica e tomografia de ossos temporais. Os dados foram analisados por meio do protocolo formulado para a obtenção dos dados, onde foram organizados e utilizado o teste estatístico Qui-quadrado. O nível de significância adotado foi de $5 \%(p \leq 0,05)$. Resultados: A microtia foi a malformação auricular mais encontrada em $65(58 \%)$ das orelhas, sendo a do tipo III a mais evidenciada em $42(64,6 \%)$ orelhas. A perda auditiva mais comum foi do tipo condutivo em $44(55,7 \%)$ e do grau severo em $41(51,9 \%)$ das orelhas. A maior parte das orelhas com microtia avaliadas por meio de tomografia também apresentavam malformação de orelha média. Conclusão: Devido à alta ocorrência de microtia e de perda auditiva nos casos estudados de EOAV, o diagnóstico, o acompanhamento e a reabilitação audiológica devem ocorrer precocemente, pois o impacto da deficiência auditiva no desenvolvimento da linguagem, pode impor a estes pacientes um obstáculo adicional para a comunicação efetiva e consequente interação social.

Palavras-chave: Espectro oculoauriculovertebral, Avaliação audiológica, Malformações auriculares, Ossos temporais. 



\section{Alves BT. Audiological findings and ear malformations in oculoauriculovertebral spectrum [dissertação]. Bauru: Hospital de Reabilitação de Anomalias Craniofaciais, Universidade de São Paulo; 2017}

Oculoauriculovertebral Spectrum (OAVS) is a congenital defect involving structures derivated from the first and second pharyngeal arches. Main clinical findings include external ear malformation, mandibular hypoplasia, epibulbar dermoid and cervical spine anomalies. External, middle and inner ear can be affected in this condition and microtia with atresia of the external auditory canal is the most common finding. Conductive and mixed hearing losses are frequently described. Objectives: To verify the presence of auricular malformation and hearing loss, investigating the possible association between them and verify the association among the temporal bone tomographic findings, the auditory function and the auricular malformations in patients diagnosed with OAVS. Material and Methods: This study was developed in the Speech and Hearing Department of the Hospital for Rehabilitation of Craniofacial Anomalies - University of São Paulo (HRCA-USP) using retrospective chart review. The sample included 72 patients with OAVS enrolled in the HRCA-USP and followed by the interdisciplinary Craniomaxillofacial Team, that presented in their records at least one audiologic assessment, description of the auricular malformation and temporal bone tomography when possible. Data were collected in a specific research protocol and statistically analyzed using the Chi-square Test with a significance level of $5 \%(p \leq 0,05)$. Results: Microtia was the most common auricular malformation, found in 65 (58\%) ears, and the most frequent type was III, occurring in 42 (64,6\%) ears. Conductive hearing loss was found in $44(55,7 \%)$ ears and severe degree in 41 (51,9\%). Most ears with microtia evaluated with tomography also presented middle ear malformation. Conclusion: Due to the high prevalence of ear malformation and hearing loss in the studied cases of OAVS, the diagnosis, follow-up and auditory rehabilitation must occur early, minimizing the impact of the hearing loss in the language development and allowing an effective oral communication and social interaction.

Keywords: Oculoauriculovertebral Spectrum, Audiologic Evaluation, Auricular Malformation, Temporal Bones. 



\section{LISTA DE TABELAS}

Tabela 1 - Distribuição dos 72 sujeitos segundo a idade, sexo e tipo de fissura labiopalatina 36

Tabela 2 - Distribuição das malformações auriculares ................................ 43

Tabela 3 - Distribuição do tipo de microtia e a lateralidade das orelhas............ 43

Tabela 4 - Distribuição do tipo e grau de perda auditiva dos sujeitos ...............44

Tabela 5 - Distribuição da audição das orelhas dos sujeitos ......................... 44

Tabela 6 - Associação entre presença e ausência de microtia e perda auditiva ......................................................................... 45

Tabela 7 - Associação entre microtia e grau de perda auditiva ....................... 45

Tabela 8 - Relação entre tipo de microtia e grau da perda auditiva ................. 46

Tabela 9 - Relação entre tipo de microtia e tipo de perda auditiva.................... 46

Tabela 10 - Associação entre microtia e tipo de perda auditiva ........................ 47

Tabela 11 - Distribuição das anormalidades do osso temporal .........................47

Tabela 12 - Associação entre presença e ausência de malformação de orelha média e microtia ........................................................................... 48

Tabela 13 - Relação entre malformação de orelha média e tipo de microtia........48

Tabela 14 - Associação entre presença e ausência de malformação de orelha média e perda auditiva ................................................... 49

Tabela 15- Relação entre malformação de orelha média e grau de perda auditiva ..................................................................... 49

Tabela 16 - Associação entre presença e ausência de malformação de orelha média e os tipos de perda auditiva.... 



\section{LISTA DE FIGURAS}

Figura 1 - Classificação dos tipos de microtia ……...........................................16

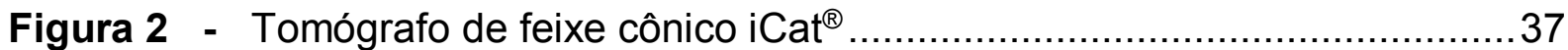





\section{LISTA DE ABREVIATURAS E SIGLAS}

AASI Aparelho de amplificação sonora individual

ATM Articulação temporomandibular

BAHA Bone Anchored Hearing Aid

CEP Comitê de Ética em Pesquisa

dBNA Decibel nível de audição

daPa Decapascal

EOAV Espectro oculoauriculovertebral

F $\quad$ Feminino

FM Frequência modulada

g.I Grau de liberdade

HRAC Hospital de Reabilitação de Anomalias Craniofaciais

$\mathrm{Hz} \quad$ Hertz

IRF Índice de reconhecimento de fala

M Masculino

MAE Meato acústico externo

N Número

OD Orelha direita

OE Orelha esquerda

OM Orelha média

p Nível de significância

RM Ressonância magnética

TC Tomografia computadorizada

USP Universidade de São Paulo

$X^{2} \quad$ Qui-quadrado 



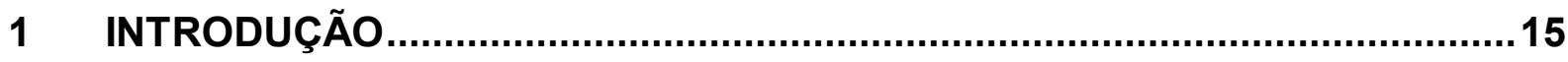

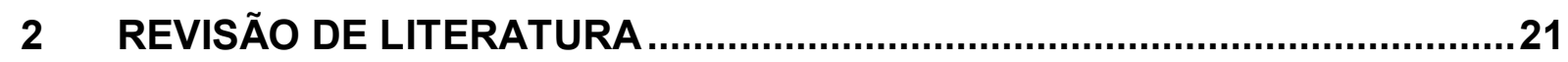

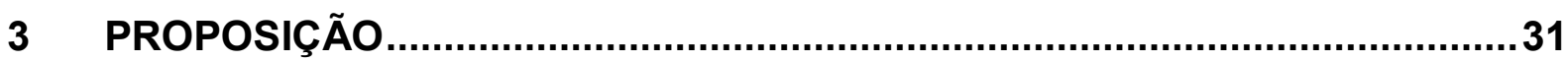

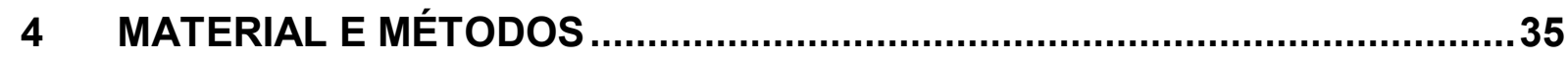

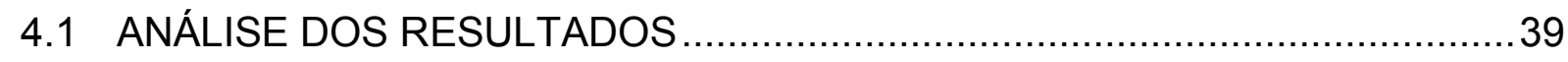

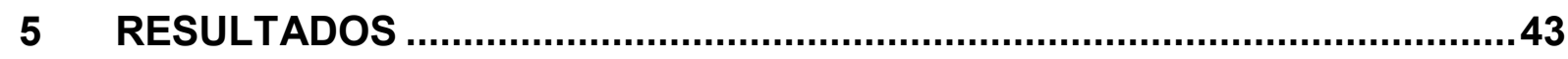

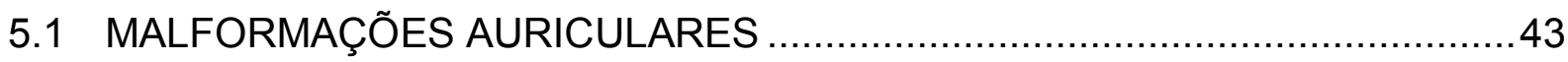

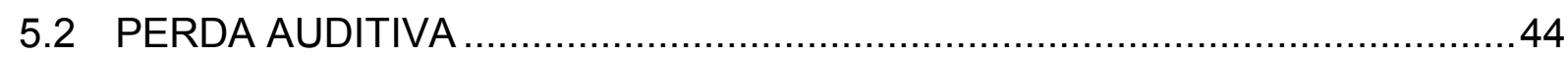

5.3 ACHADOS TOMOGRÁFICOS DE OSSOS TEMPORAIS …........................47

6 DISCUSSÃO

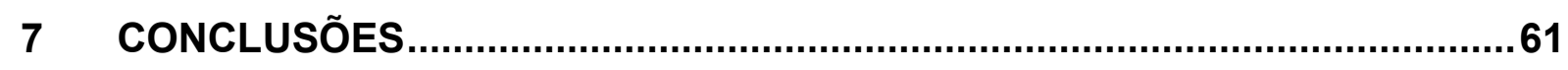

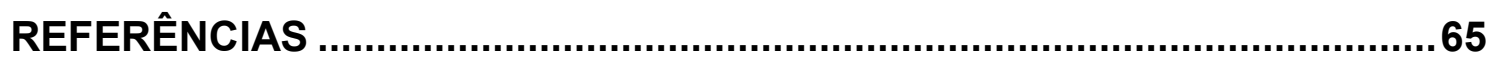

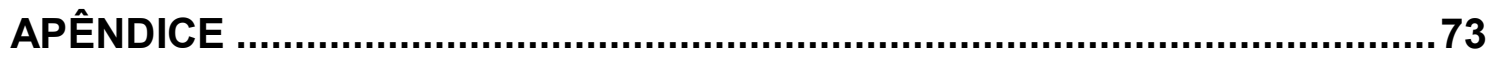

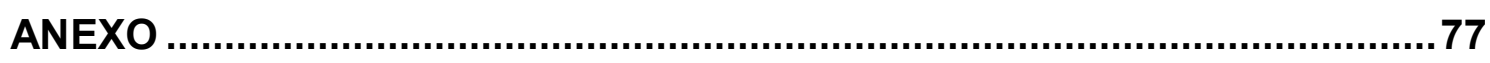



1 INTRODUÇÃO 



\section{INTRODUÇÃO}

Os avanços da fonoaudiologia e da genética como ciências complementares têm despertado cada vez mais a atenção de profissionais de ambas as áreas. Isto tem levado a um fortalecimento e conscientização quanto à necessidade de um trabalho cooperativo e multidisciplinar. Desta forma, essa parceria tem contribuído em muito, tanto para um melhor entendimento da comunicação humana e de seus distúrbios, como também, para uma melhor caracterização e elaboração de programas específicos para portadores de síndromes genéticas (ALTMANN, 2005). O trabalho do fonoaudiólogo vem se ampliando cada vez mais, sendo sua atuação limitada, não somente à avaliação e habilitação de pacientes com distúrbios da comunicação, como também, à prevenção e reabilitação dos mesmos. O seu papel, enquanto membro de uma equipe, ao estudar a base etiológica dos distúrbios da comunicação é abranger além de uma avaliação específica, a análise global de todas as manifestações apresentadas pelos pacientes (GOETZE, 2008). No Hospital de Reabilitação de Anomalias Craniofaciais da Universidade de São Paulo (HRAC/USP), o fonoaudiólogo não é somente membro integrante do tripé que compõe a base para o tratamento da fissura labiopalatina em uma equipe interdisciplinar, mas também se faz presente como um profissional atuante junto à equipe de cirurgia craniomaxilofacial, nos atendimentos a pacientes com síndromes e malformações craniofaciais. A segunda malformação congênita craniofacial mais comum depois da fissura labiopalatina, segundo Dhillon et al. (2000), é conhecida como Espectro Oculoauriculovertebral (EOAV) e, devido a sua grande frequência na rotina clínica do HRAC/USP, faz-se importante o seu estudo.

O EOAV é um defeito congênito que envolve estruturas derivadas do primeiro e segundo arcos faríngeos e tem como principais características malformação da orelha externa, hipoplasia mandibular, dermóide epibulbar e anomalia de coluna cervical. Vários termos têm sido utilizados para designar esta condição, sendo os mais comuns síndrome de Goldenhar, displasia oculoauriculovertebral, microssomia hemifacial e microssomia craniofacial. O termo EOAV foi sugerido por Cohen et al. (1989) para representar as diferentes combinações de anomalias mandibulares, auriculares, oculares e vertebrais. O fenótipo é altamente variável e as principais características clínicas incluem deformidade unilateral da orelha externa com microssomia da hemiface 
ipsilateral, dermóide epibulbar e anomalias vertebrais. Anomalias extra-craniofaciais também são descritas no EOAV, incluindo problemas cardíacos, pulmonares, gastrointestinais, geniturinários, esqueléticos e de sistema nervoso central (ROLLNICK et al., 1987).

As deformidades auriculares são variáveis e podem incluir desde apêndices préauriculares, atresia do conduto auditivo externo, anomalias de forma e tamanho do pavilhão auditivo (microtia), até a completa agenesia da orelha. Existem algumas classificações para microtia, sendo a classificação de Marx (1926) a primeira descrita na literatura. Atualmente, no Hospital de Reabilitação de Anomalias Craniofaciais é utilizada a classificação de Weerda (1988) e Hunter et al. (2009). A classificação subdivide as microtias em tipo I, II, III e IV. O tipo I tem todas as características de uma orelha normal, porém o pavilhão auricular é menor que o normal. Na tipo II, algumas estruturas anatômicas auriculares ainda são reconhecíveis e o meato acústico externo pode estar presente, ser estreito ou atrésico. Na microtia tipo III, malformação mais comum, apenas um rudimento de tecido mole está presente, com agenesia do meato acústico externo (MAE). E a tipo IV, não tem a presença de qualquer resquício do pavilhão, sendo descrita como anotia (Figura 1).
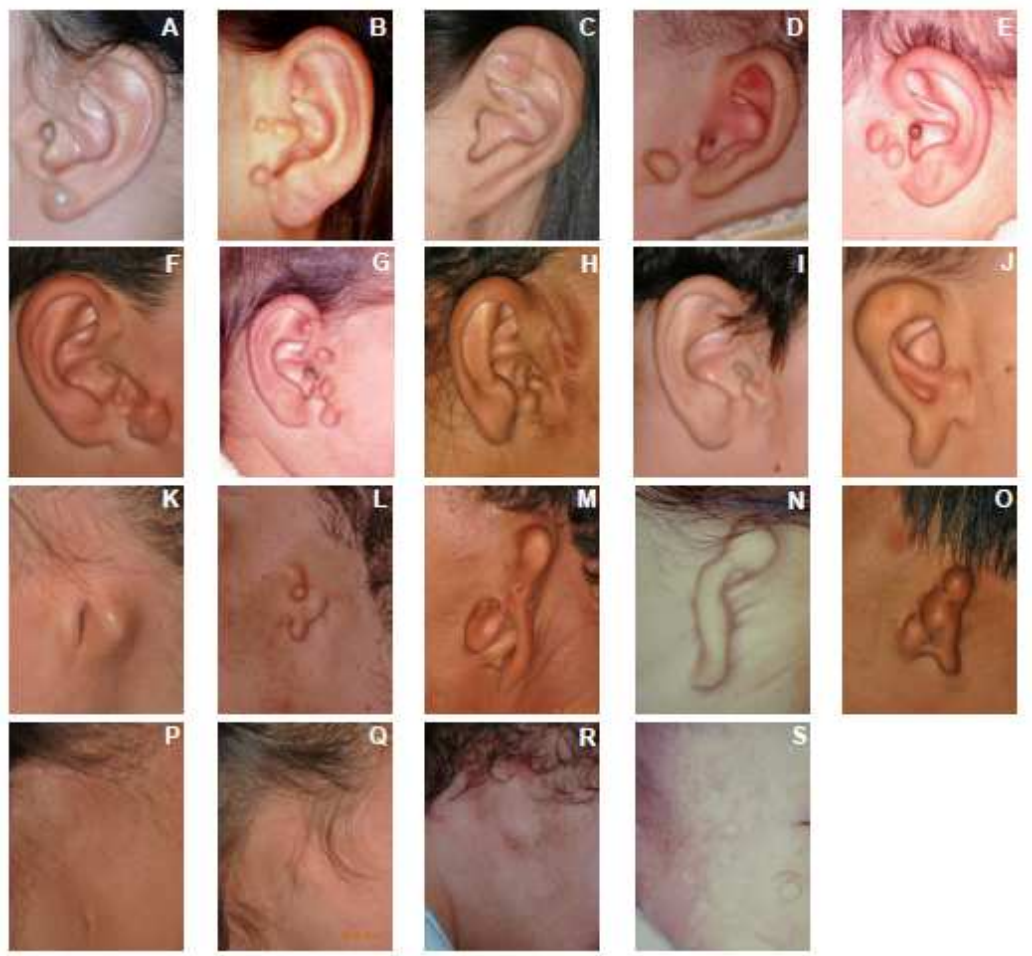

Legenda: A-E=microtia tipo I; F-G= microtia tipo II; K-O= microtia tipo III; P-S= microtia tipo IV (Anotia). Fonte: Santos JM. Espectro oculoauriculovertebral: frequência de anomalias associadas. [dissertação]. Bauru: Hospital de Reabilitação de Anomalias Craniofaciais, Universidade de São Paulo; 2015.

Figura 1 - Classificação dos tipos de microtia 
As fissuras de lábio e/ou palato podem estar associadas ao EOAV, sendo sua prevalência nesses pacientes de 7\% a 35\% (GRABB, 1965; ROLLNICK et al., 1987; VAN DER ENDE; VAN BEVER; RICHIERI-COSTA, 1993; FAN; MULLIKEN; PADWA, 2005; ROORYCK et al., 2010). Também ocorre nestes casos a assimetria do véu palatino, achado comum na rotina clínica do HRAC/USP, que pode ou não acarretar sintomas para a fala e audição. A fissura palatina conhecidamente causa distúrbios de fala, como a hipernasalidade, escape de ar nasal, deficiência de pressão intra-oral e articulações compensatórias relacionadas à disfunção velofaríngea. Além disso, o mau funcionamento da tuba auditiva pode acarretar efusão da orelha média, com otites de repetição e consequente perda auditiva (TRINDADE; SILVA FILHO, 2007). Os casos de EOAV que apresentam fissura de palato devem ser cuidadosamente manejados do ponto de vista audiológico, pois a probabilidade aumentada de otites de repetição associada à presença de microtia e malformações de orelha média compõem um quadro de alto risco para a deficiência auditiva. 

2 REVISÃO DE LITERATURA 



\section{REVISÃO DE LITERATURA}

Alguns estudos têm sido realizados no intuito de investigar a presença de anomalias de orelha no EOAV e o impacto das mesmas na função auditiva.

Rosa et al. (2011) relataram a presença de anormalidades auriculares em12 pacientes brasileiros com EOAV, envolvendo a orelha externa $(n=12)$, média $(n=8)$ e interna $(n=3)$. Oito pacientes $(67 \%)$ possuíam anormalidades de orelha média, similar à frequência de $75 \%$ descrita em outros estudos. As principais anomalias de orelha média observadas foram a opacificação do tímpano, o deslocamento e a malformação da cadeia ossicular. Embora as anomalias de orelha externa e média sejam bem conhecidas, as alterações de orelha interna (observada em $25 \%$ dos casos) são escassamente observadas e apresentam ampla variedade e diferentes graus de gravidade. Diferentemente ao observado nas orelhas externa e média, as anomalias da orelha interna ultrapassam o conceito do defeito de desenvolvimento do primeiro e segundo arcos branquiais e sugerem a inclusão de um distúrbio da migração das células da crista neural como um fator patogênico adicional no EOAV (SCHOLTZ et al., 2001). Assim, Stoll et al. (1998) colocam que, mais provavelmente, vários mecanismos patogenéticos sejam responsáveis pelo EOAV.

Sleifer et al. (2015) realizaram avaliação audiométrica em sete pacientes do sexo masculino e três do feminino com EOAV, todos com malformações de orelha. A perda auditiva condutiva foi a mais comum (10 orelhas), seguido de perda auditiva sensorioneural (cinco orelhas) e perda auditiva mista (uma orelha). O grau da perda auditiva variou de leve a moderado, com um caso de perda profunda.

Goetze et al. (2016) estudaram a audição em 10 pacientes com EOAV, dos quais todos apresentavam microtia e 5 tiveram perda de audição identificada (3 casos com perda bilateral). Perda auditiva do tipo condutiva foi encontrada em 3 pacientes, com grau variando de leve a moderado, e 2 pacientes apresentaram perda auditiva sensorioneural com grau de leve a profundo.

Mitchell et al. (2016) avaliaram a associação entre o fenótipo craniofacial e a perda auditiva em crianças com microssomia craniofacial, sendo que a microtia foi a característica mais comumente encontrada (94\%). A perda auditiva estava presente em 65 pacientes (12 com perda auditiva bilateral e 53 unilateral), sendo a perda 
auditiva do tipo condutiva a mais comum ( $73 \%$ das orelhas), seguida da perda auditiva do tipo mista (10\%), sensorioneural (1\%) e indeterminada (16\%).

Dados de prontuários de 208 pacientes chineses com EOAV e microtia atendidos num período de dois anos revelaram que o grau da perda auditiva piorou de acordo com o grau de malformação da orelha. Colesteatoma estava presente em 15 casos $(7,2 \%)$, necessitando intervenção cirúrgica. Um dado importante é que 12 das 68 orelhas contralaterais (não afetadas) apresentaram perda auditiva de grau leve a moderado (JIN et al., 2010).

Estudo retrospectivo de 70 pacientes evidenciou que medidas clínicas da hipoplasia mandibular foram preditivas dos achados tomográficos temporais, enquanto que os achados clínicos da orelha foram relacionados às anormalidades específicas do osso temporal, como hipoplasia de orelha média e fusão ou ausência dos ossículos. Não foi possível estabelecer uma relação estatisticamente significante entre os achados clínicos ou tomográficos com qualquer tipo ou grau de perda de audição (WAN et al., 2003).

Rahbar et al. (2001) realizaram estudo retrospectivo de 40 pacientes com EOAV e observaram que hipoplasia mandibular e anormalidades auriculares foram as manifestações clínicas mais comuns, presentes em 39 (97\%) e 38 pacientes (95\%), respectivamente. Perda auditiva condutiva foi encontrada em 35 pacientes $(86 \%)$ e sensorioneural em 4 (10\%). Alteração do nervo facial estava presente em 20 pacientes $(50 \%)$. Vinte pacientes apresentaram atresia aural unilateral, 12 apresentaram estenose aural unilateral e 7apresentaram anomalias bilaterais. Hipoplasia moderada ou atresia de orelha média foram encontradas em 36 (90\%) e malformação dos ossículos em 30 pacientes (75\%). Hipoplasia da janela oval foi a anormalidade de orelha interna mais comum. Concluiu-se que a severidade das características craniofaciais teve correlação significante com o grau de anormalidade de osso temporal, mas não foi notado correlação com o grau e tipo de perda auditiva.

Estudo realizado por Heike et al. (2016) com o objetivo de desenvolver uma abordagem padronizada para avaliar e descrever as características faciais de 142 indivíduos com microssomia craniofacial revelou que as características mais comumente encontradas também foram microtia $(66 \%)$ e hipoplasia mandibular (50\%).

Pegler et al. (2016) descreveram os principais achados clínicos de 41 pacientes brasileiros com displasia oculoauriculovertebral, dos quais $97,5 \%$ possuíam 
acometimento auricular. Em $31,7 \%$ dos casos, foi relatado algum grau de perda auditiva.

Cohen et al. (2017) encontraram anomalias de orelha externa (microtia ou anotia) em 74 pacientes com EOAV, sendo que 11 apresentaram malformação de orelha bilateral. A perda auditiva associada com estenose ou atresia do conduto auditivo externo foi encontrada em 69 pacientes (8 pacientes com alteração de conduto bilateral). Apêndices pré-auriculares também foram descritos em 55 pacientes.

Estudo multicêntrico envolvendo 755 pacientes com microssomia craniofacial unilateral e bilateral realizado por Caron et al. (2017) com o objetivo de analisar as anomalias craniofaciais e extra-craniofaciais revelou que $82,7 \%$ dos pacientes apresentavam anomalias auriculares, que foram graduadas de E0 a E4 (orelha normal a anotia). E3 foi encontrada na maioria dos pacientes $(64,1 \%)$.

A frequência e a relação entre perda auditiva e disfunção do nervo facial no EOAV foram estudadas por Carvalho et al. (1999). Perda auditiva ocorreu em 74 (75\%) dos 99 pacientes, com componente condutivo em 73 casos. Componente sensorioneural estava presente em 11 pacientes (11\%) e 14 pacientes foram reabilitados com aparelho de amplificação sonora. Aproximadamente $25 \%$ tiveram disfunção de nervo facial, mas somente um paciente teve paralisia facial no mesmo lado que a perda auditiva sensorioneural. Houve uma associação estatisticamente significante entre anormalidades auriculares e perda auditiva condutiva ou perda sensorioneural, porém sem significância entre a associação de microssomia hemifacial bilateral e a ocorrência de qualquer perda auditiva sensorioneural ou paralisia facial. Ausência de associação foi encontrada entre audição e disfunção do nervo facial.

Supakul, Kralik e Ho (2014) relataram um caso de EOAV com ectopia do canal auditivo externo, terminando em uma articulação temporomandibular hipoplásica e contendo estruturas ósseas com a aparência rudimentar de ossículos da orelha média.

Com os objetivos de definir a prevalência das anomalias de orelha interna em pacientes com atresia auricular e, de reconhecer padrões de anomalias desenvolvimentais nos casos de EOAV, Vrabec e Lin (2010) realizaram um estudo retrospectivo, considerando dados do exame físico, audiometria e tomografia computadorizada do osso temporal. Em 118 pacientes com atresia aural, a paralisia 
facial foi observada em $13 \%$ e anomalias de orelha interna em $22 \%$, sendo que todos aqueles com paralisia facial apresentaram alteração de orelha interna e não mostraram uma perda auditiva sensorioneural significativa. Anomalias de orelha interna bilaterais foram frequentemente encontradas, embora a atresia fosse unilateral, e envolveram predominantemente os canais semicirculares. Os autores sugerem que anomalias de orelha interna devem ser considerada uma característica comum do EOAV.

Hennersdorf et al. (2014) estudaram as mudanças no osso temporal, com ênfase especial para as anormalidades da orelha interna, em pacientes com EOAV. O estudo foi uma revisão retrospectiva de sete casos novos, além de uma série publicada anteriormente de 14 casos com síndrome de Goldenhar clinicamente diagnosticada. Além disso, os resultados foram comparados a estudos de imagem de ossos temporais da literatura. Os pacientes foram submetidos a exame otológico, estudos audiométricos e tomografia computadorizada de alta resolução (TC) ou a ressonância magnética (RM) do osso temporal, para identificar mudanças no osso temporal, especificamente em orelhas com malformações. Alterações nas orelhas externa e média foram encontradas em 19 de 21 pacientes. Sete de 21 pacientes mostraram anormalidades de orelha interna, variando de alargamento vestibular para defeitos graves como hipoplasia coclear.

Outro tipo de anomalia de orelha interna (anteversão do meato acústico interno) também foi encontrado por L'Heureux-Lebeau e Saliba (2014) em três casos de EOAV.

Estudo histopatológico dos ossos temporais de um paciente com EOAV de 10 anos de idade demonstrou deformidade auricular, atresia do canal auditivo externo e malformação da cavidade timpânica e dos ossículos. Anormalidades de estria vascular e canais semicirculares também foram demonstrados (SCHOLTZ et al., 2001).

Keogh et al. (2007) realizaram exame clínico e avaliação audiológica em pacientes com microtia uni ou bilateral com o objetivo de determinar a ocorrência de EOAV nesta casuística. EOAV foi diagnosticado em 40 pacientes com microtia (40\%), sendo a maioria com acometimento unilateral $(n=31)$. Trinta e sete pacientes demonstraram perda auditiva condutiva, e um paciente teve perda auditiva sensorioneural. Os autores concluíram que a microtia isolada representou um marcador clínico precoce para o crescimento facial assimétrico em $40 \%$ dos 
pacientes. Microtia isolada e microssomia hemifacial podem representar um espectro de expressão do mesmo fenômeno de desenvolvimento.

Nunen et al. (2014) realizaram revisão retrospectiva de 204 casos de microtia elegíveis para reconstrução auricular atendidos num período de 22 anos. Microtia unilateral do tipo lobular foi visto em 59,9\%, tipo concha em $34,4 \%$ e anotia em 5,7\%. As anomalias mais frequentes associadas à microtia foram atresia de meato acústico (76\%), apêndices pré-auriculares (30,5\%), microssomia hemifacial (27,5\%), paralisia de nervo facial $(8,3 \%)$ e cardiopatia congênita $(2,5 \%)$. Ocorrência familiar de microtia foi relatado em $2 \%$ dos pacientes do estudo e em $10 \%$ dos pacientes na literatura. $A$ maioria das anomalias congênitas associadas com microtia em pacientes holandeses pertencem ao EOAV.

Estudo multidisciplinar com 18 pacientes suecos diagnosticados com EOAV foi realizado (STRÖMLAND et al., 2007). Os achados clínicos incluíram anormalidades de orelha $(100 \%)$, malformações oculares $(72 \%)$, deformidades vertebrais $(67 \%)$, anomalias cerebrais (50\%), e cardiopatias congênitas (33\%). Defeitos funcionais consistiram em perda auditiva (83\%), deficiência visual (28\%), deficiência visual e auditiva (28\%), dificuldades alimentares (50\%), problemas na fala (53\%), deficiência intelectual (39\%) e sintomas severos de autismo (11\%). Malformação da orelha média estava presente em 12 pacientes, sendo bilateral em cinco destes. O tipo mais comum de malformação foi a fusão entre martelo e bigorna, com volume reduzido de orelha média. Malformação da orelha interna ocorreu em três pacientes, todos com defeito do órgão vestibular, sendo bilateral em um caso.

Beleza-Meireles et al. (2015) avaliaram fenótipos clínicos de 51 pacientes com EOAV e de seus pais, comparando a performance de estudos de hibridação de micro arranjos genômicos para identificar os potenciais dos loci causadores. Os resultados mostraram que $31 \%$ tinham histórico familial de EOAV. Em 55\% dos casos, as malformações foram unilaterais e, quando o envolvimento era bilateral, era assimétrico. Anormalidades auriculares estavam presentes em $92 \%$ e perda auditiva foi um achado frequente (85\%), na maioria dos casos do tipo condutivo, com alguns casos de sensorioneural ou mista. A microssomia hemifacial estava presente em $90 \%$, anomalias vertebrais em $20 \%$, anomalias encefálicas em $10 \%$, anomalias de membros em $12 \%$ e anomalias geniturinárias em $10 \%$.

Com o objetivo de caracterizar o perfil audiológico no EOAV, Brosco, Zorzetto e Richieri-Costa (2004) estudaram 30 indivíduos com este diagnóstico e verificaram 
que $34 \%$ apresentaram perda auditiva do tipo sensorioneural mista com grau variando de moderado a profundo (sete uni e três bilaterais); $13 \%$ apresentaram perda do tipo condutiva bilateral com grau de leve a severo e $3 \%$ apresentaram perda do tipo sensorioneural profundo unilateral.

Métodos cirúrgicos para a melhora da audição têm sido descritos na literatura. Dentre 11 casos de EOAV, a perda auditiva bilateral foi observada em sete e todos os pacientes necessitaram de intervenção cirúrgica que variou de procedimentos simples, como drenagem de efusão de orelha média até remoção de colesteatoma com timpanoplastia e ossiculoplastia. Dois pacientes receberam implante coclear e um necessitou implante de titânio para adaptação de BAHA (Bone Anchored Hearing Aid) (SKARZYNSKI; POROWSKI; PODSKARBI-FAYETTE, 2009).

Com relação à reabilitação auditiva, o uso de sistema de frequência modulada (FM) na escola para crianças com EOAV foi sugerido (CARRIÓN; CARDONA, 2011) mediante estudo do desempenho de uma garota com síndrome de Goldenhar, perda auditiva bilateral e flutuante e baixa aceitação da amplificação convencional. $O$ questionário do professor e estudante revelaram satisfação geral com o sistema FM, bem como um progresso significativo no desempenho acadêmico.

O Hospital de Reabilitação de Anomalias Craniofaciais da Universidade de São Paulo é referência no tratamento interdisciplinar das malformações congênitas e desenvolve seu trabalho há 49 anos, contando atualmente com mais de 100.000 indivíduos matriculados. Inicialmente voltado ao tratamento das fissuras labiopalatinas, hoje atende uma variedade de síndromes e malformações craniofaciais complexas associadas ou não às fissuras orofaciais, bem como as deficiências auditivas. A fissura labiopalatina é a anomalia craniofacial congênita de maior ocorrência, seguida pelo EOAV. Devido ao número expressivo de indivíduos com este diagnóstico em nossa instituição, faz-se pertinente o estudo multidisciplinar dos mesmos. Atualmente estão cadastrados aproximadamente 400 indivíduos com diagnóstico de EOAV no HRAC-USP, incluindo casos com outras anomalias como, por exemplo, o EOAV com anomalia radial. Um estudo desenvolvido no Setor de Genética Clínica do HRAC-USP (PITTOLI, 2010) teve por objetivo analisar e descrever a morfologia da mandíbula, com ênfase na articulação temporomandibular (ATM), e investigar orelha média e interna em indivíduos com EOAV. Alta frequência de anomalias mandibulares $(87,5 \%)$ e de orelha média $(96,1 \%)$ foi verificada. Relações preditivas entre a ocorrência e a gravidade das anomalias mandibulares 
com o acometimento da orelha externa e com o acometimento das orelhas média e interna foram observadas.

Mediante os achados morfológicos, faz-se importante o estudo de sua relação com os aspectos funcionais, evidenciando a importância de se investigar a audição. Hipotetiza-se que, quanto maior o grau de malformação da orelha, mais comprometida estará a acuidade auditiva. 

3 PROPOSIÇÃO 



\section{PROPOSIÇÃO}

\subsection{Objetivo Geral:}

- Estudar a relação entre malformações auriculares e perda auditiva em indivíduos com espectro oculoauriculovertebral.

\subsection{Objetivos Específicos:}

- Verificar o tipo de malformação auricular;

- Verificar a presença de perda auditiva, classificando-a quanto ao tipo e ao grau;

- Verificar a possível associação entre o tipo de malformação auricular e os achados audiológicos;

- Verificar a possível associação entre os achados tomográficos de ossos temporais com a audição. 

4 MATERIAL e MÉtodos 



\section{MATERIAL E MÉTODOS}

O trabalho recebeu a aprovação pelo Comitê de Ética em Pesquisa (CEP $n^{\circ}$ 2.096.030) do Hospital de Reabilitação de Anomalias Craniofaciais (HRAC), da Universidade de São Paulo (USP) (Anexo A).

Primeiramente foi solicitada a profissionais da craniomaxilofacial do HRACUSP uma lista de pacientes que apresentavam EOAV com documentação de imagem, o que permitiu a análise de 173 prontuários de pacientes cadastrados. Foi realizado o cálculo amostral estatístico, sendo sugerido pela profissional estatística uma amostra de 72 pacientes. Assim, a casuística incluiu pacientes atualmente acompanhados pela equipe de cirurgia craniomaxilofacial do hospital, que já apresentavam em sua documentação clínica o exame de tomografia, e pelo menos uma avaliação audiológica. A faixa etária avaliada foi de cinco a 55 anos. Somente 20 pacientes foram incluídos no estudo dos achados tomográficos, devido à falta de documentação disponível.

Critérios de inclusão:

1. Apresentar diagnóstico clínico de EOAV;

2. Apresentar avaliação audiológica prévia;

3. Apresentar dados quanto ao tipo de malformação auricular ou registro fotográfico antes de intervenções cirúrgicas para que seja possível a classificação.

Critérios de exclusão:

1. Pacientes com quadros genéticos distintos do EOAV.

A tabela 1 demonstra a distribuição dos sujeitos da pesquisa quanto a idade, sexo e tipo de fissura labiopalatina. 
Tabela 1 - Distribuição dos 72 sujeitos segundo a idade, sexo e tipo de fissura labiopalatina

\begin{tabular}{|c|c|c|c|c|c|c|c|c|c|}
\hline \multirow{3}{*}{$\begin{array}{l}\text { Faixa etária } \\
\text { (anos) }\end{array}$} & & & \multicolumn{6}{|c|}{ Tipos de Fissuras Labiopalatinas } & \multirow{3}{*}{ Tota } \\
\hline & \multicolumn{2}{|c|}{ Palato íntegro } & \multicolumn{2}{|c|}{$\begin{array}{c}\text { Pré- } \\
\text { forame }\end{array}$} & \multicolumn{2}{|c|}{ Transforame } & \multicolumn{2}{|c|}{$\begin{array}{l}\text { Pós- } \\
\text { forame }\end{array}$} & \\
\hline & $M$ & $\mathbf{F}$ & $\mathbf{M}$ & $\mathbf{F}$ & M & $\mathbf{F}$ & M & $\mathbf{F}$ & \\
\hline 5 a 7 & 9 & 6 & 1 & 0 & 2 & 0 & 2 & 2 & 22 \\
\hline 8 a 10 & 9 & 6 & 0 & 0 & 2 & 2 & 0 & 2 & 21 \\
\hline 11 a 20 & 4 & 6 & 1 & 1 & 2 & 0 & 6 & 0 & 20 \\
\hline 21 e acima & 0 & 2 & 0 & 0 & 2 & 2 & 1 & 2 & 9 \\
\hline Total & 22 & 20 & 2 & 1 & 8 & 4 & 9 & 6 & 72 \\
\hline
\end{tabular}

Legenda: $F=$ feminino; $M=$ masculino; $m=m e ̂ s$

O número de pacientes que apresentaram fissura no palato foi $27(37,5 \%)$, distribuídos na tabela 1.

Dos 72 pacientes com EOAV, 62 pacientes apresentaram microtia (59 com microtia unilateral e três com microtia bilateral), sendo que o lado afetado por microtia também apresentava hipoplasia mandibular.

O presente estudo foi realizado utilizando dados clínicos das Seções de Fonoaudiologia, Otorrinolaringologia, Ortodontia e Genética do HRAC-USP, onde os casos de EOAV têm sido rotineiramente avaliados e acompanhados, e envolveu análise retrospectiva de fontes secundárias de dados. Os dados obtidos foram transcritos no protocolo de pesquisa (Apêndice A).

Os dados coletados foram relativos a:

1. Identificação do paciente;

2. Diagnóstico genético e sinais clínicos;

3. Antecedentes e indicações cirúrgicas;

4. Avaliações otorrinolaringológicas;

5. Avaliação audiológica mais recente: audiometria tonal liminar, logoaudiometria e imitanciometria;

6. Exames de imagem: tomografia de ossos temporais;

7. Tipo de malformação auricular, mediante avaliação clínica e tomográfica.

Os exames de imagem (tomografia de ossos temporais) foram previamente realizados em algum momento da rotina clínica do paciente, utilizando um tomógrafo 
de feixe cônico (iCatß) da instituição (Figura 2), e foram analisados em parceria com profissional da área de Otorrinolaringologia.

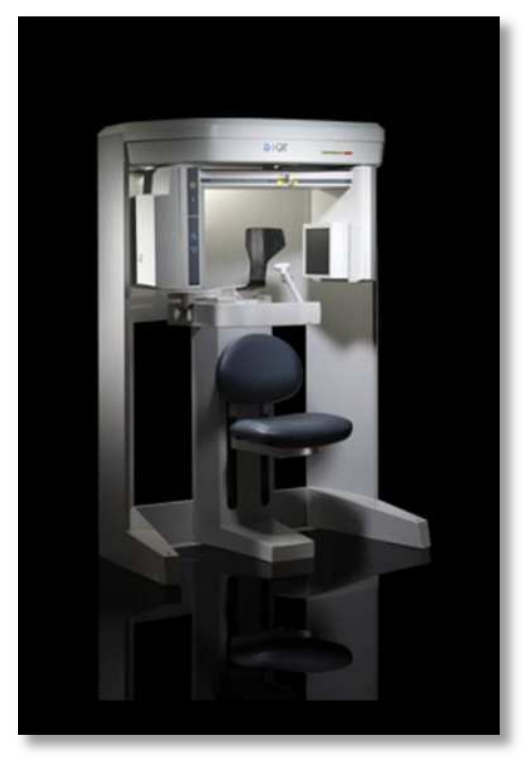

Fonte: iCat ${ }^{\circledR}$. Award-Winning Cone Beam 3D Dental Imaging. c2016.

Figura 2 - Tomógrafo de feixe cônico iCat ${ }^{\circledR}$

As avaliações audiológicas foram realizadas no Setor de Fonoaudiologia do HRAC-USP pela fonoaudióloga responsável da equipe craniomaxilofacial, utilizando os seguintes equipamentos: audiômetros Kamplex AD27 Diagnostic Audiometer e Madsen Midimate 622 Clinical/DiagnosticAudiometer e imitanciômetros AT235 Interacoustics, AZ7-R Interacoustics e MT10/P10 Interacoustics, e composta de:

I. História Clínica: Mediante entrevista padronizada aplicada na rotina do Setor de Fonoaudiologia, na qual são coletadas informações sobre o desenvolvimento auditivo e de linguagem, bem como sua condição clínica na data do exame. Para isso, é verificada a presença de queixa auditiva (otite, otalgia, otorréia, hipoacusia e outros) atual e pregressa, uso de medicamentos e cirurgias otológicas realizadas ou indicadas. Também se considera o estado de saúde geral, a presença de doenças, estado gripal atual ou recente.

II. Inspeção otológica: Consiste na visualização do MAE e da membrana timpânica, usualmente realizada anterior a avaliação audiológica para descartar a presença de corpo estranho, tubo de ventilação, cerúmen, 
otorreia, perfuração timpânica e outros, que podem comprometer a realização dos exames e/ou a confiabilidade dos resultados. Para isso, é utilizado um otoscópio de fibra ótica, modelo standard N $2.5 \mathrm{~V}$, da marca Heine.

III. Medidas de imitância acústica: Exame que permite avaliar as condições funcionais do sistema tímpano-ossicular. Composto pela timpanometria e pela pesquisa do reflexo do músculo estapédio, fornece medidas importantes para a correta interpretação dos demais achados audiológicos. As curvas timpanométricas obtidas são classificadas em cinco tipos, de acordo com Jerger (1970):

- Tipo A: Indicativa de funcionamento adequado da orelha média, com valores de compliância variando entre 0,3 e 1,3 ml e pressão do pico de máxima compliância próximo a $0 \mathrm{daPa}$, sendo considerados como normais valores entre -100 e $+100 \mathrm{daPa}$ em adultos e entre -150 e +90 daPa em crianças.

- Tipo Ar: Indicativa de rigidez sistema tímpano-ossicular, com compliância menor que $0,3 \mathrm{ml}$ e pressão próxima a 0 daPa.

- Tipo Ad: Indicativa de disjunção de cadeia ossicular ou membrana timpânica monomérica, com compliância maior que $1,3 \mathrm{ml}$ e pressão próxima de 0 daPa.

- Tipo B: Indicativa de secreção na orelha média, sem pico de máxima compliância.

- Tipo C: Indicativa de disfunção tubária, com compliância dentro da normalidade e pico deslocado para as pressões negativas.

IV. Audiometria Tonal Liminar e Logoaudiometria: Este procedimento é utilizado para avaliar a audição em nível periférico por meio da obtenção de limiares auditivos, de 250 a $8000 \mathrm{~Hz}$ por via aérea e de 500 a $4000 \mathrm{~Hz}$ por via óssea, quando necessário. Quando não for possível realizar o exame em toda esta faixa de frequência, por falta de colaboração ou atenção do paciente, são priorizados os limiares nas frequências de 500 , 1000, 2000 e $4000 \mathrm{~Hz}$. O limite de normalidade dos limiares tonais considerado foi de até $20 \mathrm{dBNA}$, conforme proposto por Davis e Silverman em 1970. Para crianças até sete anos de idade, o limite de normalidade é 
de 15dBNA (NORTHERN; DOWNS, 1984). Estes mesmos valores foram acatados para a classificação do grau de perda auditiva, considerando a média tonal das frequências de 500, 1000 e $2000 \mathrm{~Hz}$. O tipo de perda auditiva obedeceu a classificação: condutiva, sensorioneural e mista. Para o grau foi seguido: leve, moderada, severa ou profunda. (SANTOS; RUSSO, 1993).

A logoaudiometria foi composta pelo índice de reconhecimento de fala (IRF), utilizando lista de 25 palavras monossilábicas foneticamente balanceadas (SANTOS; RUSSO, 1993) e limiar de reconhecimento de fala (LRF), com palavras dissílabas selecionadas aleatoriamente, da lista utilizada no IRF.

Os exames foram realizados em cabina acústica da marca VIBRASOM Tecnologia acústica Ltda, em sala acusticamente preparada, utilizando audiômetros devidamente calibrados por pessoal qualificado.

Não foram incluídos nesse estudo dados de outras avaliações audiológicas (p.ex. avaliação eletrofisiológica, emissões otoacústicas, etc), uma vez que não são rotineiramente realizadas nesta faixa etária.

\subsection{Análise de dados}

Os dados foram organizados e analisados no que se refere a análise descritiva das malformações auriculares, perda auditiva e achados tomográficos de ossos temporais. O teste estatístico utilizado foi o Qui-quadrado nas relações entre microtia e presença, grau e tipo de perda auditiva, e nas malformações de orelha média com presença de microtia, presença e tipo de perda auditiva, sendo adotado o nível de significância de $5 \%(p \leq 0,05)$. 

5 Resultados 



\section{RESULTADOS}

\subsection{Malformações auriculares}

Das 144 orelhas analisadas, 79 (54,86\%) apresentaram malformações auriculares, que consistiram em microtia e/ou apêndices pré-auriculares. A tabela 2 demonstra a distribuição das malformações auriculares.

Tabela 2 - Distribuição das malformações auriculares

\begin{tabular}{cc}
\hline MALFORMAÇÃO AURICULAR & $\begin{array}{c}\text { ORELHA } \\
\mathbf{N}(\%)\end{array}$ \\
\hline APÊNDICE PRÉ - AURICULAR & $37(33,0)$ \\
MICROTIA & $65(58,0)$ \\
\hline TOTAL & $102(100)$ \\
\hline
\end{tabular}

Legenda: $\mathrm{N}=$ número, $(\%)=$ porcentagem

Alguns sujeitos apresentaram mais que uma malformação na mesma orelha

Tendo em vista a maior prevalência de microtia, elaborou-se a tabela 3 quanto ao tipo e lateralidade. A análise mostrou que a microtia tipo III à direita foi a de maior ocorrência.

Tabela 3- Distribuição do tipo de microtia e a lateralidade das orelhas

\begin{tabular}{cccc}
\hline \multirow{2}{*}{ MICROTIA } & \multicolumn{2}{c}{ LATERALIDADE } & TOTAL \\
& OD & OE & N (\%) \\
\cline { 2 - 3 } & $7(10,8)$ & $4(6,1)$ & $11(16,9)$ \\
TIPO I & $6(9,2)$ & $3(4,6)$ & $9(13,8)$ \\
TIPO II & $22(33,8)$ & $20(30,8)$ & $42(64,6)$ \\
TIPO III & $2(3,1)$ & $1(1,5)$ & $3(4,6)$ \\
TIPO IV & $37(56,9)$ & $28(43,1)$ & $65(100)$ \\
\hline TOTAL &
\end{tabular}

Legenda: $\mathrm{OD}=$ orelha direita, $\mathrm{OE}=$ orelha esquerda, $\mathrm{N}=$ número, $(\%)=$ porcentagem 


\subsection{Perda auditiva}

Em relação à audição, considerando-se todas as orelhas ( $n=144), 65(45,13 \%)$ apresentaram audição normal e 79 (54,86\%) apresentaram perda auditiva. A Tabela 4 demonstra a distribuição do tipo e grau de perda auditiva presente na amostra.

Tabela 4 - Distribuição do tipo e grau de perda auditiva dos sujeitos

\begin{tabular}{cccccc}
\hline \multirow{2}{*}{$\begin{array}{c}\text { TIPO DE PERDA } \\
\text { AUDITIVA }\end{array}$} & \multicolumn{4}{c}{ GRAU DE PERDA AUDITIVA } & \multirow{2}{*}{ TOTAL } \\
\cline { 2 - 5 } N $(\%)$ & LEVE & MODERADO & SEVERO & PROFUNDO & N $\%)$ \\
\hline CONDUTIVA & $12(15,2)$ & $17(21,5)$ & $15(19,0)$ & $0(0,0)$ & $44(55,7)$ \\
MISTA & $0(0,0)$ & $6(7,6)$ & $26(32,9)$ & $1(1,2)$ & $33(41,8)$ \\
SENSORIONEURAL & $0(0,0)$ & $0(0,0)$ & $0(0,0)$ & $2(2,5)$ & $2(2,5)$ \\
\hline TOTAL & $12(15,2)$ & $23(29,1)$ & $41(51,9)$ & $3(3,8)$ & $79(100)$ \\
\hline
\end{tabular}

Legenda: $\mathrm{N}=$ número, $(\%)=$ porcentagem

Considerando-se as orelhas com microtia, 62 (95,3\%) apresentaram perda auditiva e três $(4,6 \%)$ audição normal. A Tabela 5 demonstra a audição da amostra das orelhas.

Tabela 5 - Distribuição da audição das orelhas dos sujeitos

\begin{tabular}{|c|c|c|c|}
\hline \multirow[t]{2}{*}{ ORELHA } & \multicolumn{2}{|c|}{$\begin{array}{c}\text { AUDIÇÃO } \\
\text { N (\%) }\end{array}$} & \multirow{2}{*}{$\begin{array}{c}\text { TOTAL } \\
\text { N (\%) }\end{array}$} \\
\hline & NORMAL & PERDA AUDITIVA & \\
\hline SEM MICROTIA & $63(43,7)$ & $16(11,1)$ & $79(54,8)$ \\
\hline COM MICROTIA & $2(1,4)$ & $63(43,7)$ & $65(45,1)$ \\
\hline TOTAL & $65(45,1)$ & $79(54,8)$ & $144(100)$ \\
\hline
\end{tabular}

Legenda: $\mathrm{N}=$ número, $(\%)=$ porcentagem

Dentre as orelhas sem microtia e com perda auditiva, $14(87,5 \%)$ orelhas, correspondiam a orelhas contralaterais àquelas com microtia, sendo que duas orelhas tinham apêndices pré-auriculares associados. Desses pacientes, nove apresentavam fissura palatina, sendo mais um fator para a perda auditiva na orelha sem a malformação auricular.

Verificou-se associação entre as variáveis microtia e perda auditiva, demonstrando que, quando há presença de microtia a perda auditiva está frequentemente presente, conforme demonstrado na tabela 6. 
Tabela 6 - Associação entre presença e ausência de microtia e perda auditiva

\begin{tabular}{|c|c|c|c|}
\hline \multirow{2}{*}{ MICROTIA } & \multicolumn{2}{|c|}{$\begin{array}{c}\text { PERDA AUDITIVA } \\
\mathrm{N}(\%) \\
\end{array}$} & \multirow{2}{*}{ VALOR DE $p$} \\
\hline & AUSENTE & PRESENTE & \\
\hline AUSENTE & $63(79,7)$ & $16(20,3)$ & $p<0,001$ \\
\hline PRESENTE & $2(3,1)$ & $63(96,9)$ & \\
\hline \multicolumn{4}{|c|}{$X^{2}=81,579$} \\
\hline \multicolumn{4}{|c|}{ g.I = 1} \\
\hline
\end{tabular}

Legenda: $\mathrm{N}=$ número, $(\%)=$ porcentagem

No que se refere à presença de microtia e o grau de perda auditiva, devido à baixa ocorrência de perda auditiva de grau profundo $(n=3)$, para fins estatísticos, este número foi acrescido à categoria perda severa, sendo os resultados demonstrados na tabela 7 .

Tabela 7 - Associação entre microtia e grau de perda auditiva

\begin{tabular}{|c|c|c|c|c|c|}
\hline \multirow{3}{*}{$\begin{array}{l}\text { MICROTIA } \\
\text { AUSENTE }\end{array}$} & \multicolumn{4}{|c|}{$\begin{array}{c}\text { GRAU DE PERDA AUDITIVA } \\
\mathrm{N}(\%) \\
\end{array}$} & \multirow{2}{*}{ VALOR DE $p$} \\
\hline & NORMAL & LEVE & MODERADO & $\begin{array}{l}\text { SEVERO + } \\
\text { PROFUNDO }\end{array}$ & \\
\hline & $63(79,7)$ & $9(11,4)$ & $7 \quad(8,9)$ & $0 \quad(0,0)$ & $0<0001$ \\
\hline PRESENTE & $2(3,1)$ & $3(4,6)$ & $16(24,6)$ & $44(67,7)$ & \\
\hline
\end{tabular}

Legenda: $\mathrm{N}=$ número, $(\%)=$ porcentagem

$\mathrm{Na}$ análise entre o tipo de microtia e o grau da perda auditiva foi evidenciado que, quanto maior o grau de comprometimento da estrutura da orelha externa (microtia), maior o grau da perda auditiva (Tabela 8). 
Tabela 8 - Relação entre tipo de microtia e grau da perda auditiva

\begin{tabular}{ccccccc}
\hline \multirow{2}{*}{ MICROTIA } & \multicolumn{5}{c}{ GRAU DA PERDA AUDITIVA } & N (\%) \\
\cline { 2 - 6 } & NORMAL & LEVE & MODERADO & SEVERO & PROFUNDO & N (\%) \\
\cline { 2 - 6 } TIPO I & $2(3,1)$ & $2(3,1)$ & $6(9,2)$ & $1(1,5)$ & $0(0,0)$ & $11(16,9)$ \\
TIPO II & $1(1,5)$ & $0(0,0)$ & $3(4,6)$ & $5(7,7)$ & $0(0,0)$ & $9(13,8)$ \\
TIPO III & $0(0,0)$ & $0(0,0)$ & $6(9,2)$ & $\mathbf{3 3}(\mathbf{5 0 , 8 )}$ & $3(4,6)$ & $42(64,6)$ \\
TIPO IV & $0(0,0)$ & $0(0,0)$ & $1(1,5)$ & $2(3,1)$ & $0(0,0)$ & $3(4,6)$ \\
\hline TOTAL & $3(4,6)$ & $2(3,1)$ & $16(24,6)$ & $\mathbf{4 1 ( 6 3 , 1 )}$ & $3(4,6)$ & $65(100)$ \\
\hline
\end{tabular}

Legenda: $\mathrm{N}=$ número, $(\%)=$ porcentagem

A ocorrência e a diferença estatisticamente significante entre os tipos de perda auditiva e de microtia são demonstradas nas tabelas 9 e 10, respectivamente. Devido ao baixo índice de perda auditiva do tipo sensorioneural $(n=2)$, para fins de aplicação de teste estatístico, este número foi acrescido à perda auditiva do tipo mista. $\mathrm{Na}$ maioria das orelhas com microtia, houve uma ocorrência semelhante de perdas do tipo condutiva e mista e nas orelhas sem microtia, predominou a audição normal. (Tabela 10).

Tabela 9 - Ocorrência dos diferentes tipos de perda auditiva em relação ao tipo de microtia

\begin{tabular}{|c|c|c|c|c|c|}
\hline \multirow{2}{*}{ MICROTIA } & \multicolumn{4}{|c|}{$\begin{array}{c}\text { TIPO DE PERDA AUDITIVA } \\
\mathbf{N}(\%) \\
\end{array}$} & \multirow{2}{*}{$\begin{array}{l}\text { TOTAL } \\
\text { N (\%) }\end{array}$} \\
\hline & NORMAL & CONDUTIVA & MISTA & SENSORIONEURAL & \\
\hline TIPO I & $2(3,1)$ & $7(10,7)$ & $2(3,1)$ & $0(0,0)$ & $11(16,9)$ \\
\hline TIPO II & $1(1,5)$ & $3(4,6)$ & $5(7,7)$ & $0(0,0)$ & $9(13,8)$ \\
\hline TIPO III & $0(0,0)$ & $17(26,1)$ & $23(35,4)$ & $2(3,1)$ & $42(64,6)$ \\
\hline TIPO IV & $0(0,0)$ & $1(1,5)$ & $2(3,1)$ & $0(0,0)$ & $3(4,6)$ \\
\hline TOTAL & $3(4,6)$ & $28(43,1)$ & $32(49,2)$ & $2(3,1)$ & $65(100)$ \\
\hline
\end{tabular}

Legenda: $\mathrm{N}=$ número, $(\%)=$ porcentagem 
Tabela 10 - Análise da relação entre microtia e tipo de perda auditiva

\begin{tabular}{|c|c|c|c|c|}
\hline \multirow{2}{*}{ MICROTIA } & \multicolumn{3}{|c|}{$\begin{array}{c}\text { TIPO DE PERDA AUDITIVA } \\
\mathbf{N}(\%)\end{array}$} & \multirow{2}{*}{ VALOR DE $p$} \\
\hline & NORMAL & CONDUTIVA & $\begin{array}{c}\text { MISTA + } \\
\text { SENSORIONEURAL }\end{array}$ & \\
\hline AUSENTE & $63(79,7)$ & $15 \quad(19,0)$ & $1 \quad(1,3)$ & $p<0,001$ \\
\hline PRESENTE & $2(3,1)$ & $29 \quad(44,6)$ & $34 \quad(52,3)$ & \\
\hline \multicolumn{5}{|c|}{$X^{2}=92,327$} \\
\hline & & $g . I=2$ & & \\
\hline
\end{tabular}

Legenda: $\mathrm{N}=$ número, $(\%)=$ porcentagem

\subsection{Achados tomográficos de ossos temporais}

Dos 20 sujeitos que apresentaram documentação dos achados tomográficos, foi observado que $22(55 \%)$ orelhas apresentaram alguma malformação de orelha média (OM), sendo $13(59 \%)$ à direita e nove (40,9\%) à esquerda. Mastóide hipopneumatizada e não pneumatizada, orelha média com ausência de espaço e orelha média ausente, cadeia ossicular malformada e ausente, alteração de nervo facial e obliteração de janela oval foram as malformações encontradas. A tabela 11 demonstra a distribuição dessas alterações.

Tabela 11 - Distribuição das anormalidades do osso temporal

\begin{tabular}{cc}
\hline ANORMALIDADES DO OSSO TEMPORAL/MALFORMAÇÃO DE OM & $\begin{array}{c}\text { ORELHA } \\
\text { N }(\%)\end{array}$ \\
MASTOIDE HIPOPNEUMATIZADA & $15(34,9)$ \\
MASTOIDE NÃO PNEUMATIZADA & $1(2,3)$ \\
OM COM AUSÊNCIA DE ESPAÇO & $11(25,6)$ \\
OM AUSENTE & $2(4,6)$ \\
CADEIA OSSICULAR MALFORMADA & $4(9,3)$ \\
CADEIA OSSICULAR AUSENTE & $6(13,9)$ \\
ALTERAÇÃO DE NERVO FACIAL & $3(6,9)$ \\
OBLITERAÇÃO DE JANELA OVAL & $1(2,3)$ \\
\hline & $43(100)$ \\
\hline
\end{tabular}


A presença de microtia e a microtia do tipo III foram as maiores ocorrências nas malformações de orelha média, conforme demonstram as tabelas 12 e 13, respectivamente.

Tabela 12 - Associação entre presença e ausência de malformação de orelha média e microtia

\begin{tabular}{|c|c|c|c|}
\hline \multirow{2}{*}{$\begin{array}{c}\text { MALFORMAÇÃO DE } \\
\text { OM }\end{array}$} & \multicolumn{2}{|c|}{$\begin{array}{c}\text { MICROTIA } \\
\text { N (\%) }\end{array}$} & \multirow{2}{*}{ VALOR DE $p$} \\
\hline & AUSENTE & PRESENTE & \\
\hline AUSENTE & $16(88,9)$ & $2(11,1)$ & \multirow{2}{*}{$p<0,001$} \\
\hline PRESENTE & $5(22,7)$ & $17(77,3)$ & \\
\hline \multicolumn{4}{|c|}{$X^{2}=14,826$} \\
\hline \multicolumn{4}{|c|}{$g . I=1$} \\
\hline
\end{tabular}

Legenda: $\mathrm{OM}=$ orelha média, $\mathrm{N}=$ número, $(\%)=$ porcentagem

Tabela 13 - Relação entre malformação de orelha média e tipo de microtia

\begin{tabular}{|c|c|c|c|c|c|}
\hline \multirow{2}{*}{$\begin{array}{c}\text { MALFORMAÇÃO DE } \\
\text { OM }\end{array}$} & \multicolumn{4}{|c|}{$\begin{array}{l}\text { TIPO DE MICROTIA } \\
\text { N (\%) }\end{array}$} & \multirow{2}{*}{$\begin{array}{c}\text { TOTAL } \\
\text { N (\%) }\end{array}$} \\
\hline & TIPO I & TIPO II & TIPO III & TIPO IV & \\
\hline AUSENTE & $0(0,0)$ & $0(0,0)$ & $2(10,5)$ & $0(0,0)$ & $2(10,5)$ \\
\hline PRESENTE & $5(26,3)$ & $4(21,1)$ & $7(36,8)$ & $1(5,3)$ & $17(89,5)$ \\
\hline TOTAL & $5(26,3)$ & $4(21,1)$ & $9(47,3)$ & $1(5,3)$ & $19(100)$ \\
\hline
\end{tabular}

Legenda: $\mathrm{OM}=$ orelha média, $\mathrm{N}=$ número, $(\%)=$ porcentagem

A presença de perda auditiva e a perda auditiva do tipo mista foram achados significantes em orelhas com malformação de orelha média, conforme demonstrado nas tabelas 14 e 16, respectivamente, sendo o grau de moderado a severo o mais encontrado. A audição dentro dos padrões de normalidade foi encontrada para a maioria das orelhas sem malformação de orelha média (Tabela 15). 
Tabela 14 - Associação entre presença e ausência de malformação de orelha média e perda auditiva

\begin{tabular}{|c|c|c|c|}
\hline \multirow{2}{*}{$\begin{array}{c}\text { MALFORMAÇÃO DE } \\
\text { OM }\end{array}$} & \multicolumn{2}{|c|}{$\begin{array}{c}\text { PERDA AUDITIVA } \\
\text { N (\%) } \\
\end{array}$} & \multirow{2}{*}{ VALOR DE $p$} \\
\hline & AUSENTE & PRESENTE & \\
\hline AUSENTE & $11 \quad(61,1)$ & $7 \quad(38,9)$ & \\
\hline PRESENTE & $(13,6)$ & $19(86,4)$ & $p=0,005$ \\
\hline \multicolumn{4}{|c|}{$X^{2}=7,832$} \\
\hline \multicolumn{4}{|c|}{$g . I=1$} \\
\hline
\end{tabular}

Legenda: $\mathrm{OM}=$ orelha média, $\mathrm{N}=$ número, $(\%)=$ porcentagem

Tabela 15 - Relação entre malformação de orelha média e grau de perda auditiva

\begin{tabular}{ccccccc}
\hline \multirow{2}{*}{$\begin{array}{c}\text { MALFORMAÇÃOO } \\
\text { DE OM }\end{array}$} & NORMAL & LEVE & MODERADO & SEVERO & PROFUNDO & \multirow{2}{*}{\begin{tabular}{c} 
TOTAL \\
\cline { 2 - 6 }
\end{tabular}} \\
\cline { 2 - 6 } & & & $1(2,5)$ & $1(2,5)$ & $0(0,0)$ & $18(45,0)$ \\
\hline AUSENTE & $11(27,5)$ & $5(12,5)$ & $1(\%)$ & $1(2,5)$ & $22(55,0)$ \\
PRESENTE & $3(7,5)$ & $1(2,5)$ & $7(7,5)$ & $10(25,0)$ & $1(2,5)$ & $40(100)$ \\
\hline TOTAL & $14(35,0)$ & $6(15,0)$ & $8(20,0)$ & $11(27,5)$ & $1(2,5)$ & \\
\hline
\end{tabular}

Legenda: $\mathrm{OM}=$ orelha média, $\mathrm{N}=$ número, $(\%)=$ porcentagem

Tabela 16 - Associação entre presença e ausência de malformação de orelha média e os tipos de perda auditiva

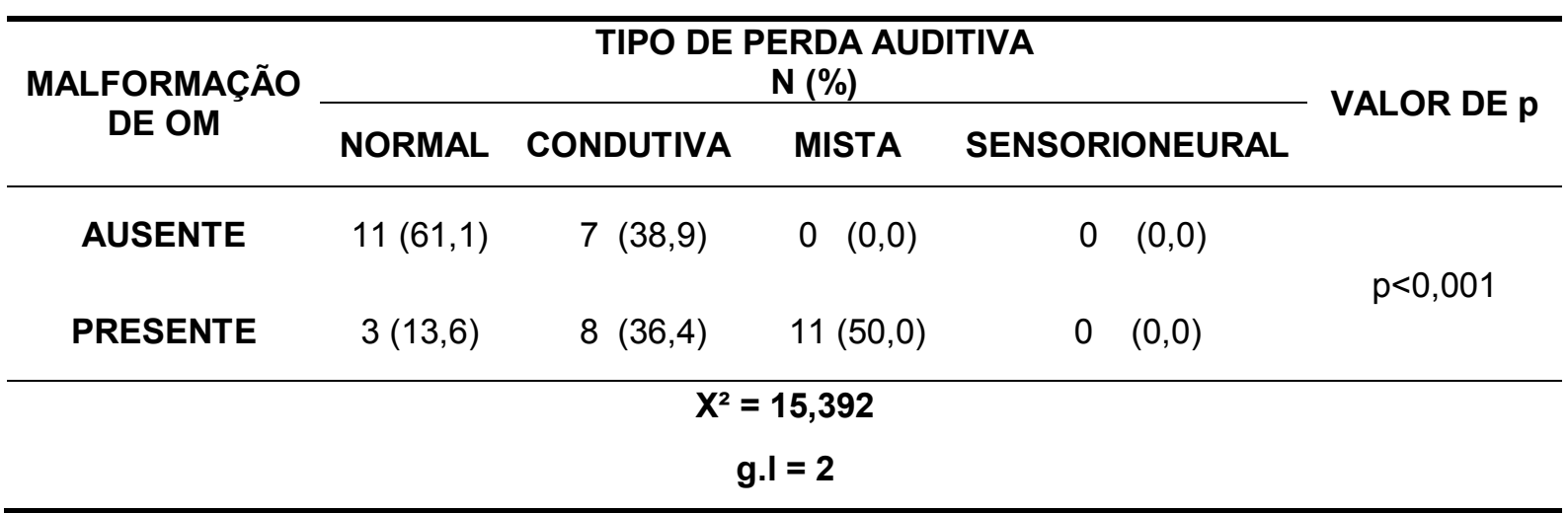

Legenda: $\mathrm{OM}=$ orelha média, $\mathrm{N}=$ número, $(\%)=$ porcentagem 

6 DIscussÃo 



\section{DISCUSSÃO}

$\mathrm{Na}$ literatura consultada, alguns trabalhos anteriormente realizados (HEIKE et al., 2016; MITCHELL et al., 2016; PEGLER et al., 2016; CARON et al., 2017), anteriormente realizados, mostraram a alta ocorrência de malformações auriculares nos pacientes com EOAV, semelhante a presente investigação, na qual esses achados clínicos foram frequentes. Pesquisa conduzida por Pittoli (2010) na mesma instituição desse estudo, com casos de anomalias de $1^{\circ}$ e $2^{\circ}$ arcos faríngeos, também mostrou que a maioria deles apresentavam malformação de orelha externa. Ainda, corroborando com os achados publicados (RAHBAR et al., 2001; JIN et al., 2010; ROSA et al., 2011; BELEZA-MEIRELES et al., 2015; GOETZE et al., 2016; COHEN et al., 2017; STROMLAND et al., 2017), a microtia foi a principal alteração encontrada nos pacientes com EOAV (Tabela 2). Como há controvérsia quanto a um critério mínimo para o seu diagnóstico, visto que em alguns estudos somente a presença de microtia isolada já é considerada uma forma leve dentro do espectro da condição (ROLLNICK et al., 1987; KEOGH et al., 2007), ou somente a presença de apêndice pré-auricular ou microssomia hemifacial (JIN et al., 2010), se faz importante identificar a presença e diferenciar as malformações auriculares, visto que sua ocorrência é frequente nesses pacientes, auxiliando assim, no diagnóstico genético, audiológico e processo de reabilitação.

Tendo em vista que a maior parte da casuística do presente estudo foi composta por casos de EOAV unilateral à direita, o lado direito também foi o mais afetado pela microtia (Tabela 3 ). O número baixo de três pacientes com microtia bilateral na casuística pode ser justificado pelo perfil dos pacientes que realizam acompanhamento audiológico no Setor de Fonoaudiologia do HRAC-USP da área de Fissuras e Anomalias Craniofaciais, onde não se realiza reabilitação audiológica. $O$ acompanhamento audiológico para os casos com microtia bilateral, por usualmente requerer o uso de dispositivos eletrônicos de amplificação sonora, ocorre em outro serviço (Divisão de Saúde Auditiva). Na literatura também é descrito que o lado mais afetado pela microtia é o lado direito (KEOGH et al., 2007; JIN et al., 2010; PITTOLI, 2010; NUNEN et al., 2014; CARON et al., 2017). Alguns estudos também tiveram em sua amostra a maior parte de pacientes com acometimento auricular unilateral 
(PITTOLI, 2010; NUNEN et al., 2014; HEIKE et al., 2016), mostrando que a incidência de pacientes com EOAV unilaterais são mais frequentes do que os bilaterais.

Neste estudo, apesar de todos os tipos de microtia terem sido encontrados (Tabela 3), a predominância do tipo III corrobora com os achados dos estudos de Wan et al. (2003); Keogh et al. (2007); Jin et al. (2010); Rosa et al. (2011); Caron et al. (2017). Na sequência, ocorreram a microtia tipo II e por último a do tipo I. Pittoli (2010) encontrou a microtia tipo II como mais frequente, seguido do tipo III e da microtia tipo IV e Caron et al. (2017) relataram que a microtia tipo I foi mais frequente que a tipo II, divergindo do presente estudo. A classificação do tipo de microtia pode indicar os diferentes graus de comprometimento da orelha externa. Assim, deve-se ter em mente, como demonstrado no presente estudo (Tabela 3) que, quanto maior o comprometimento auricular (microtia tipo III), pior o grau da perda auditiva (severo) diagnosticada (Tabela 7).

A perda auditiva, em seus diferentes graus e tipos, presente nas orelhas com microtia deste estudo (Tabelas 5, 8 e 9) mostrou associação significante entre a presença de microtia com a perda auditiva (Tabela 6), confirmando o anteriormente relatado nos trabalhos de Carvalho et al. (1999); Stromland et al. (2007); BelezaMeireles et al. (2015); Mitchell et al. (2016); Cohen et al. (2017). No entanto, o estudo de Pegler et al. (2016) mostrou que apenas $31,7 \%$ de sua população com displasia OAV apresentaram perda auditiva.

A investigação da audição é necessária e essencial para os indivíduos com EOAV, não somente na orelha em que há a presença de malformação auricular, que pareceu influenciar negativamente a audição, mas também, na orelha contralateral a ela (Tabela 5), apoiando o estudo de Jin et al. (2010). Neste sentido, é importante considerar a população em estudo, uma vez que alguns casos apresentavam fissura de palato (Tabela 1), que causa problemas relacionados à audição devido ao mau funcionamento da tuba auditiva acarretando em efusão da orelha média, com otites de repetição e consequente perda auditiva (TRINDADE; SILVA FILHO, 2007). As fissuras de lábio e/ou palato podem estar associadas ao EOAV de 7 a 35\% (GRABB, 1965; ROLLNICK et al., 1987; VAN DER ENDE; VAN BEVER; RICHIERI-COSTA, 1993; FAN; MULLIKEN; PADWA, 2005; ROORYCK et al., 2010), apoiando os achados desse estudo, que encontrou uma frequência de 37,5.

A perda auditiva de grau severo (Tabela 4) encontrado no presente estudo, vem fornecer um dado diferente do observado em parte da literatura (WAN et al., 2003; 
SLEIFER et al., 2015; GOETZE et al., 2016), que relata o grau moderado como sendo o de maior frequência em indivíduos com EOAV. No entanto, o grau moderado foi o segundo mais frequente neste estudo. O trabalho realizado por Brosco et al. (2004) mostrou que houve variação de seu grau nos diferentes tipos de perdas auditivas diagnosticados, variando de leve a severo nos casos de perda do tipo condutiva, moderado a profundo nas perdas mistas e, leve a severo nas perdas sensorioneurais, achados similares foram encontrados do presente estudo (Tabela 4).

Em ordem decrescente de ocorrência, os tipos de perdas auditivas condutiva, mista e sensorioneural foram os observados (Tabelas 4, 9 e 10), mostrando que há relação significante entre a presença da microtia com o tipo de perda auditiva. Este achado vem apoiar os estudos em sua maioria, que mostram a perda auditiva condutiva como a mais encontrada nesses pacientes (CARVALHO et al., 1999; RAHBAR et al., 2001; WAN et al., 2003; KEOGH et al., 2007; STROMLAND et al., 2007; BELEZA-MEIRELES et al., 2015; SLEIFER et al., 2015; MITCHELL et al., 2016). No entanto, Brosco et al. (2004) encontraram uma ocorrência maior de perda auditiva do tipo mista, divergindo do presente estudo.

$\mathrm{Na}$ literatura, alguns estudos analisaram a orelha média nos casos de EOAV, com presença de algum tipo de malformação variando de 28,8\% a 90\% (RAHBAR et al., 2001; SCHOLTZ et al., 2001; WAN et al., 2003; STROMLAND et al., 2007; PITTOLI, 2010; ROSA et al., 2011), concordando com os achados do presente estudo de $55 \%$. No presente estudo, a mastoide hipopneumatizada e a orelha média com ausência de espaço foram as malformações mais encontradas (Tabela 11). No entanto, Stromland et al. (2007) e Rosa et al. (2011) relataram que as principais anomalias observadas foram a malformação de cadeia ossicular e a falta de aeração da mastoide, discordando com o presente estudo, onde essas anomalias também foram encontradas, porém em menor relevância.

São poucos os estudos com EOAV na literatura que analisaram a orelha média, e a maioria deles refere-se a relatos de casos. Ressalta-se, no presente estudo, a importância de realizar o exame tomográfico de ossos temporais, uma vez que o mesmo permite a visualização de suas estruturas, pois com a presença de malformação auricular associada a agenesia do MAE, não é possível avaliar as condições funcionais do sistema tímpano-ossicular por meio das medidas de imitância acústica. 
No presente estudo mostrou-se que há relação significante entre a presença de microtia e malformação de orelha média (Tabela 12), como também visto por Rahbar et al. (2001); Wan et al. (2003); Stromland et al. (2007); Pittoli (2010) e Rosa et al. (2011). O estudo também evidenciou a relação da malformação de orelha média com o tipo de microtia, pois a mesma ocorreu em todos os tipos de microtia, porém com maior frequência na microtia tipo III (Tabela 13). Isso demonstra que a malformação auricular não traz somente o comprometimento estético, mas também o funcional, no qual, não somente afeta a condução do som, pela presença de perda condutiva, mas também a recepção sonora, nas perdas mista e sensorioneural presentes e o grau da perda auditiva, onde foi evidenciado que há relação significante entre a presença de malformação de orelha média com a presença e com o tipo de perda auditiva, não sendo citado na literatura dados quanto à esse aspecto específico. (Tabelas 14, 15 e 16).

A audição é um canal sensorial essencial para a comunicação humana (DANIELI et al., 2011). A perda auditiva tem impacto negativo na qualidade de vida do indivíduo, visto que o déficit auditivo pode acarretar em dificuldades na compreensão da fala, podendo sofrer consequências psicossociais, além de incapacidade de se proteger em situações de risco de vida. Por essas razões, o restabelecimento dos limiares de audibilidade torna-se necessário, através da reabilitação auditiva (PICININI et al., 2017), sobretudo nos pacientes com EOAV, no qual foi demonstrado nesse estudo e na literatura, que a maioria dos sujeitos podem apresentar perda auditiva.

O fonoaudiólogo é o profissional responsável na identificação da perda de audição, classificando-a quanto ao tipo e ao grau, essencial para a reabilitação desses pacientes. Assim, por meio de uma eficiente avaliação audiológica é possível verificar a presença de perda auditiva associada às malformações auriculares, permitindo que a equipe de profissionais faça a melhor opção para o processo reabilitador, seja ele cirúrgico, uso de dispositivos eletrônicos e/ou acompanhamento audiológico.

Quando a escolha é a indicação cirúrgica, a mesma é realizada a partir dos 6 ou 7 anos de idade (FETTERMAN; LUXFORD, 1997). No caso dos dispositivos eletrônicos, a adaptação precoce do AASI é fundamental para favorecer a estimulação auditiva e o desenvolvimento da aquisição da fala e linguagem. No entanto, uma vez que o uso do AASI (aparelho de amplificação sonora individual) por via aérea não é possível nos casos de agenesia do MAE, (WAZEN; CARUSO; TJELLSTROM, 1998), 
como é o caso dos $42(64,6 \%)$ pacientes deste estudo, o BAHA (Bone Anchored Hearing Aid), dispositivo eletrônico por condução óssea tipo tiara, é uma alternativa que apresenta vantagens sobre os aparelhos de condução óssea convencionais e, indicado para os pacientes com acometimento bilateral da microtia com agenesia do MAE. A decisão do tipo de amplificação a ser utilizado cabe aos profissionais responsáveis pelo caso, em conjunto com o sujeito e familiares (PACCOLA; FERNANDES; MONDELLI, 2013). 

7 CONCLUSÕES 



\section{CONCLUSÕES}

Os pacientes com EOAV avaliados no presente estudo apresentaram, em sua maioria, malformações auriculares e presença de perda auditiva, como uma relação significativa entre esses dois fatores. As malformações auriculares presentes foram apêndices pré-auriculares e microtia, sendo essa a mais evidenciada, e a do tipo III de maior ocorrência nos pacientes. A perda auditiva mais comumente encontrada foi do tipo condutivo e do grau severo. Quando presente a microtia, a mesma apresentou associação com a perda auditiva, mostrando que quanto maior o comprometimento auricular, pior o grau da perda auditiva diagnosticada.

A maior parte das orelhas com microtia avaliadas por meio de tomografia também apresentavam malformação de orelha média. Em orelhas sem malformação de pavilhão auricular, também foi identificada perda auditiva e malformação de orelha média, a maioria destas sendo em orelhas contralaterais às que apresentavam microtia.

O diagnóstico, o acompanhamento e a reabilitação audiológica em casos de EOAV devem ocorrer precocemente, mesmo quando não é detectada malformação da orelha externa no exame clínico. O impacto da deficiência auditiva no desenvolvimento da linguagem é assunto de amplo conhecimento, impondo a estes pacientes um obstáculo adicional para a comunicação efetiva e consequente interação social.

O tratamento ideal para estes casos deve ocorrer em centros especializados e envolver equipe interdisciplinar. Destaca-se o papel do fonoaudiólogo, médico otorrinolaringologista e cirurgião craniofacial no que diz respeito ao tratamento das malformações de orelha e perdas de audição por elas ocasionadas. 




\section{REFERÊNCIAS}

ALTMANN, E.B.C. Fissuras labiopalatais. Editora: Pró-fono, $4^{\circ}$ edição, São PauloSP, 2005

BELEZA-MEIRELES, A. et al. Oculo-auriculo-vertebral spectrum: Clinical and molecular analysis of 51 patients. European Journal of Medical Genetics 58 (2015) 455-465.

BROSCO, K.C.; ZORZETTO, N.L.; RICHIERI-COSTA, A. Perfil audiológico de indivíduos portadores da síndrome de Goldenhar. Rev Bras Otorrinolaringol. V.70, n.5, 645-9, set./out. 2004.

CARON, C.J.J.M. et al. Craniofacial and extracraniofacial anomalies in craniofacial macrosomia: A multicenter study of 755 patients. Journal of CranioMaxillo-Facial Surgery 45, 2017; 1302 e 1310.

CARRIÓN, M.; CARDONA, G. Goldenhar syndrome with moderate hearing loss: An FM system in a school environment. International Journal of Pediatric Otorhinolaryngology Extra 6, 2011; 178-181.

CARVALHO, G.J. et al. Auditory and Facial Nerve Dysfunction in Patients with Hemifacial Microsomia. Arch Otolaryngol Head Neck Surg. 1999; 125: 209-212.

COHEN, M.M.JR.; ROLLNICK, B.R.; KAYE, C.I. Oculoauriculovertebral spectrum: an updated critique. Cleft Palate J. 1989; 26(4):276-86.

COHEN, N. et al. Maxillofacial features and systemic malformations in expanded spectrum Hemifacial Microsomia. Am J Med Genet Part A. 2017.

DANIELI, F. et al. Avaliação do nível de satisfação de usuários de aparelhos de amplificação sonora individuais dispensados pelo Sistema Único de Saúde. Rev Soc Bras Fonoaudiol. 2011; 16(2):152-9.

DAVIS, H.; SILVERMAN, R.S. Hearing and deafness. Nova York: Rinehart \& Wiston; 1970.

DHILLON, M. et al. Hemifacial Microsomia: a clinic radiological report of three cases. Journal of Oral Science, Chengdu, v. 52, n. 2, p. 319-24, 2010. 
FAN, W.S.; MULLIKEN, J.B.; PADWA, B.L. An association between hemifacial microsomia and facial clefting. J Oral Maxillofac Surg. 2005; 63(3):330-4.

FETTERMAN, B.L.; LUXFORD, W.M. The rehabilitation of conductive hearing impairment. Otolaryngol Clin North Am. 1997; 30(5):783-801. PMid: 9295254.

GOETZE, T.B. et al. Fonoaudiologia e genética: avanços nos estudos em indivíduos com fissuras labiopalatinas. IX Salão de Iniciação Científica PUCRS. 2008.

GOETZE, T.B. et al. Hearing Characterization in Oculoauriculovertebral Spectrum: A Prospective Study with 10 Patients. Am J Med Genet Part A 173A:309314. 2016.

GRABB, W.C. The first and second branchial arch syndrome. Plast Reconstr Surg. 1995; 36: 485-508.

HEIKE, C.L. et al. Characterizing Facial Features in Individuals with Craniofacial Microsomia: A Systematic Approach for Clinical Research. Birth Defects Research (Part A) 106:915-926. 2016.

HENNERSDORF, F. et al. Temporal Bone Changes in Patients With Goldenhar Syndrome With Special Emphasis on Inner Ear Abnormalities. Otology \& Neurotology, Vol. 35, No. 5, 2014.

HUNTER, A. et al. Elements of morphology: Standart terminology for the ear. Am J Med Genet A. 2009; 149(1):40-60.

JERGER, J. Clinical experience with impedance audiometry. Arch Otolaryngol. 1970; 92(4):311-24.

JIN, L. et al. Clinical analysis based on 208 patients with microtia (especially reviewed oculo - auriculo - vertebral spectrum, hearing test, CT scan). The Turkish Journal of Pediatrics 2010; 52: 582-587.

$\mathrm{KEOGH}$, I. et al. Isolated Microtia as a Marker for Unsuspected Hemifacial Microsomia. Arch Otolaryngol Head Neck Surg. 2007; 133(10):997-1001.

L'HEUREUX-LEBEAU, B.; SALIBA, I. Anteverted internal auditory canal as an inner ear anomaly in patients with craniofacial microsomia. International Journal of Pediatric Otorhinolaryngology 78. 2014; 1551-1553. 
MARX, H. Die Missbildungen des Ohres. In: Denker A, Kahler O, Hrsg. Handbuch der HNO-Heilkunde. Bd. 6/1. Berlin: Springer; 1926. S. 131-69.

MITCHELL, R.M. et al. Hearing Loss in Children with Craniofacial Microsomia. Cleft Palate-Craniofacial Journal, Month 0000, Vol. 00 No. 00. 2016.

NORTHEN, J.L.; DOWS, M.P. Hearing in Children. $3^{a} . e d$. Williams \& Wilkins, Baltimore; 1984. p. 89.

NUNEN, D.P.F. et al. Microtia in the Netherlands: Clinical characteristics and associated Anomalies. International Journal of Pediatric Otorhinolaryngology 78. 2014; 954-959.

PACCOLA, E.C.M.; FERNANDES, J.C.; MONDELLI, M.F.C.G. Amplification by bone conduction in congenital malformations: patient benefits and satisfaction. Brazilian Journal of Otorhinolaryngology 79 (3) Maio/Junho 2013.

PEGLER, J.R.M. et al. Clinical description of 41 Brazilian patients with oculoauriculo-vertebral dysplasia. Rev Assoc Med Bras 2016; 62(3):202-206.

PICININI, T.A. et al. Restriction of social participation and satisfaction of hearing aids - post-adaptation study. Audiol Commun Res. 2017; 22: e1830.

RAHBAR, R. et al. Craniofacial, Temporal Bone, and Audiologic Abnormalities in the Spectrum of Hemifacial Microsomia. Arch Otolaryngol Head Neck Surg. 2001; 127: 265-271.

ROLLNICK, B.R. et al. Oculoauriculovertebral dysplasia and variants: phenotypic characteristics of 294 patients. Am J Med Genet 1987; 26(2): 361-75.

ROORYCK, C. et al. Array-CGH analysis of a cohort of 86 patients with oculoauriculovertebral spectrum. Am J Med Genet A. 2010; 152A(8):1984-9.

Rosa, R.F.M. et al. Ear abnormalities in patients with oculo-auriculo-vertebral spectrum (Goldenhar syndrome). Braz J Otorhinolaryngol. 2011; 77(4):455-60.

SANTOS, J.M. Espectro oculoauriculovertebral: frequência de anomalias associadas [dissertação]. Bauru: Hospital de Reabilitação de Anomalias Craniofaciais, Universidade de São Paulo; 2015. 
SANTOS, T.M.M; RUSSO, I.C.P. A prática da audiologia clínica. São Paulo: Cortez; 1993.

SCHOLTZ, A.W. et .al. Goldenhar's Syndrome: Congenital Hearing Deficit of Conductive or Sensorineural Origin? Temporal Bone Histopathologic Study. Otology \&Neurotology22:501-505 (c) 2001, Otology \& Neurotology, Inc.

SKARZYNSKI, H.; POROWSKI, M.; PODSKARBI-FAYETTE, R. Treatment of otological features of the oculoauriculovertebral dysplasia (Goldenhar syndrome). International Journal of Pediatric Otorhinolaryngology 73. 2009; 915-921.

SLEIFER, P. et al. Audiological Findings in Patients with Oculo-AuriculoVertebral Spectrum. Int Arch Otorhinolaryngol 2015; 19:5-9.

STOLL, C. et al. A family with dominant oculoauriculovertebral spectrum. Am J Med Genet 1998; 78(4):345-9.

STRÖMLAND, K. et al. Oculo-Auriculo-Vertebral Spectrum: Associated Anomalies, Functional Deficits and Possible Developmental Risk Factors. American Journal of Medical Genetics Part A 143A:1317-1325; 2007.

SUPAKUL, N.; KRALIK, S.F.; HO, C.H. Ectopic external auditory canal and ossicular formation in the oculo-auriculo-vertebral spectrum. Pediatr Radiol. 2015; 45:1099-1102.

TRINDADE, I.E.K.; SILVA FILHO, O.G. Fissuras labiopalatinas: uma abordagem interdisciplinar. São Paulo: Santos Editora; 2007.

VAN DEN ENDE, J.J.; VAN BEVER, Y.; RICHIERI-COSTA, A. The OAV-spectrum and associated anomalies in 77 patients. Rev Bras Genet. 1993; 16(3):811-17.

VENDRAMINI-PITTOLI, S. Investigação radiológica e tomográfica da mandíbula de indivíduos com anomalias de $1^{\circ}$ e $2^{\circ}$ arcos faríngeos [tese]. Bauru: Hospital de Reabilitação de Anomalias Craniofaciais, Universidade de São Paulo; 2010.

VENDRAMINI, S.; RICHIERI-COSTA, A.; GUION-ALMEIDA, M.L. Oculoauriculovertebral spectrum with radial defects: a new syndrome or an extension of the oculoauriculovertebral spectrum? Report of fourteen Brazilian cases and review of the literature. European Journal of Human Genetics. 2007; 15, 411-421.

VRABEC, J.T.; LIN, J.W. Inner Ear Anomalies in Congenital Aural Atresia. Otology \& Neurotology, Vol. 31, No. 9, 2010. 
WAN, J. ET AL. Clinical, Radiological, and Audiological Relationships in Hemifacial Microsomia. Annals of Plastic Surgery Volume 51 / Number 2 / August 2003.

WAZEN, J.J.; CARUSO, M.; TJELLSTROM, A. Long-term results with the titanium bone-anchored hearing aid: the U.S. experience. Am J Otol. 1998; 19(6):737-41.

WEERDA, $\mathrm{H}$. Classification of congenital deformities of the auricle. Facial Plast Surg. 1988; 5(5):385-8. 

APÊNDICE 



\section{APÊNDICE A - PROTOCOLO DE COLETA DE DADOS}

\section{Identificação}

No.

D.N: RG:

Nome:

Fissura: ( ) não ( ) sim - tipo:

Diagnóstico genético:

Malformação auricular: ( ) ausente ( ) unilateral - tipo:

( ) bilateral - OD tipo: OE tipo:

Intervenções cirúrgicas: ( ) não ( ) sim:

\section{Avaliação audiológica}

Data: ___ I__ Idade na avaliação:___ _ _ Avaliações prévias: ( ) não ( ) sim

( ) Acompanhamento audiológico

Finalidade do exame:
( ) controle
( ) pré-cirúrgico
( ) pós-cirúrgico

\subsection{Anamnese}

( ) Sem queixas

( ) Queixas:

\subsection{Avaliação}

2.2.1 Timpanometria - tipo de curva segundo classificação de Jerger (1970):
( ) Tipo A
( ) Tipo B
( ) OD
( ) $\mathrm{OE}$
( ) Tipo C
( ) OD
( ) $\mathrm{OE}$
( ) Tipo Ad
( ) OD
( ) $\mathrm{OE}$
( ) Tipo Ar
( ) OD
( ) OE
( ) Não realizada
( ) OD
( ) OE
( ) OD
( ) OE

2.2.2 Pesquisa dos reflexos estapédicos - em dB:

\begin{tabular}{|l|l|l|l|l|l|}
\hline \hline \multicolumn{1}{|c|}{ OD } & $500 \mathrm{~Hz}$ & $1000 \mathrm{~Hz}$ & $2000 \mathrm{~Hz}$ & $4000 \mathrm{~Hz}$ & Não pesquisado \\
\hline Contralateral & & & & & \\
\hline Ipsilateral & & & & & \\
\hline
\end{tabular}

\begin{tabular}{|l|l|l|l|l|l|}
\hline \multicolumn{1}{|c|}{$\mathrm{OE}$} & $500 \mathrm{~Hz}$ & $1000 \mathrm{~Hz}$ & $2000 \mathrm{~Hz}$ & $4000 \mathrm{~Hz}$ & Não pesquisado \\
\hline Contralateral & & & & & \\
\hline Ipsilateral & & & & & \\
\hline
\end{tabular}


2.2.3 Audiometria tonal liminar - limiares em dB (Santos e Russo 1993):

\begin{tabular}{|l|l|l|l|l|l|}
\hline VO & $500 \mathrm{~Hz}$ & $1000 \mathrm{~Hz}$ & $2000 \mathrm{~Hz}$ & $3000 \mathrm{~Hz}$ & $4000 \mathrm{~Hz}$ \\
\hline OD & & & & & \\
\hline OE & & & & & \\
\hline
\end{tabular}

\begin{tabular}{|l|l|l|l|l|l|l|l|l|}
\hline VA & $250 \mathrm{~Hz}$ & $500 \mathrm{~Hz}$ & $1000 \mathrm{~Hz}$ & $2000 \mathrm{~Hz}$ & $3000 \mathrm{~Hz}$ & $4000 \mathrm{~Hz}$ & $6000 \mathrm{~Hz}$ & $8000 \mathrm{~Hz}$ \\
\hline OD & & & & & & & & \\
\hline OE & & & & & & & \\
\hline
\end{tabular}

2.2.4 Conclusão - classificação segundo Davis e Silverman (1970):
( ) Audição dentro da normalidade
( ) OD
( ) OE
( ) Perda auditiva leve
( ) OD
( ) OE
( ) Perda auditiva moderada
( ) OD
( ) $\mathrm{OE}$
( ) Perda auditiva severa
( ) OD
( ) $\mathrm{OE}$
( ) Perda auditiva profunda
( ) OD
( ) OE

Tipo:

$\begin{array}{lll}\text { ( ) Perda auditiva condutiva } & \text { ( ) OD } & \text { ( ) OE } \\ \text { ( ) Perda auditiva mista } & \text { ( ) OD } & \text { ( ) OE } \\ \text { ( ) Perda auditiva sensorioneural } & \text { ( ) OD } & \text { ( ) OE }\end{array}$

Equipamento utilizado na avaliação audiológica:

Imitanciômetro:

$\begin{array}{lll}\text { ( ) AT235 ( ) AZ7R ( ) MT10 } & \text { ( ) }\end{array}$

Audiômetro:

( ) AD 27 ( ) Midimate 622 ( ) IVRA

\section{Tomografia}


ANEXO 



\section{ANEXO A - APROVAÇÃO NO COMITÊ DE ÉTICA EM PESQUISA}

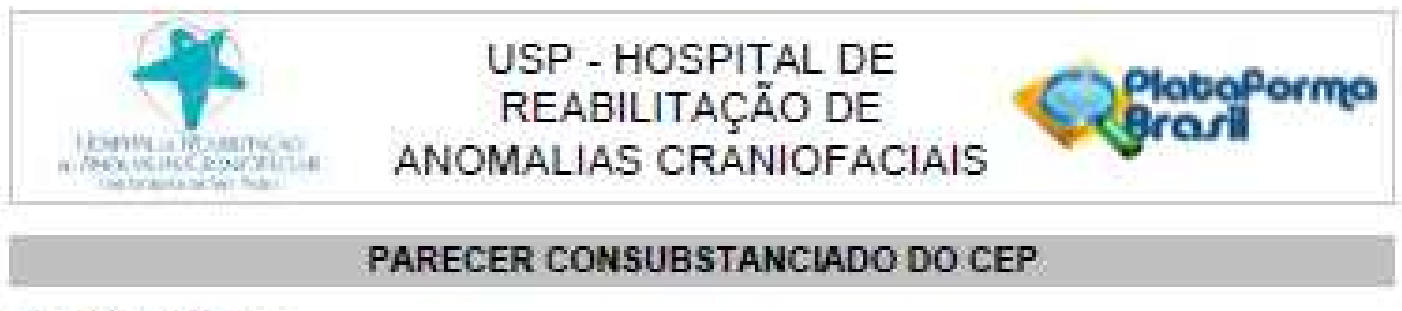

\section{DADOS DA EMENDA}

Titulo da Pesquilsa: Achados audioiogicos e matomaçles aurlaulares no Espectro Ccuicaurculovertebral

Pesqulsador: Bruna Tozzen Aves

Area Tematica:

Versas: 3

CAAE: 60843716.1 .0000 .5441

Institulçăo Proponente: Hospita de Reaclitaç5o de Anomalas Cranlotacials da usP

Patrocinador Principal: Financiamento Proprio

\section{DADOS DOPARECER}

Numero do Parecer. 2.096.030

\section{Apresentaça do Projeto:}

Trata-se de projeto de Dissertaçás da aluna Bruna Tozzett Alves, sob ontentaçăo da Prota. Dra. Marisa Ribelro Feniman e coorientaçăo da Dra. Mellssa Zattonl Antonell. O estudo sera reallzado, utilizando-se dados clinicos do Setor de Fonoaudiologla e Ortodonta do HRAC-USP, onde os casos de EOAV tem sido rotherramente avallados e acompanhados, e envolvera analise retrospectva de prontuarios. Os dados coletados serdo relatvos a:

1. Identincaç50 do paciente, data de nascimento, procedencla, idade na matricula.jdade na uttima avaliaçato audiologica, dagnostico geneoco, sinals cilnicos; 2 . Antecedentes e indicaçJes dirurgicas; 3 . Avalaçbles otortinolaningoiogicas; 4. Avalaç5̆o audiologloa mals recente: audiometrla tonal liminar, logoaudiometria e Imitanclometria; 5. Uso de ampincaçato sonora; 6. Achados da tomografia de mandioula e de ossos temporals; 7. Tipo de malformaçăo auricular, medlante avallaçăo cinica e tomografica; 8 . Tipo de maitormaçăo mandbular, medlante avaliaç5o clinica e tomograflca.

Os exames $d$ imagen (tomografla da mandibula) foram previamente realzados no setor de Ortodonta do HRAC-USP e serăo analsados em parcerla com profissional da area. Estes exames estăo atuaimente sendo utllizados em dlssertaça de mestrado na area de Ortodontla (aprovaçato CEP no.1.373.379). As Imagens relacionadas a0s ossos temporals seráo anallsadas juntamente

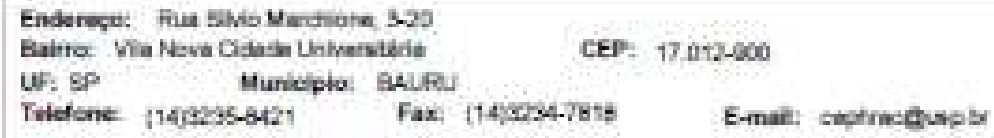




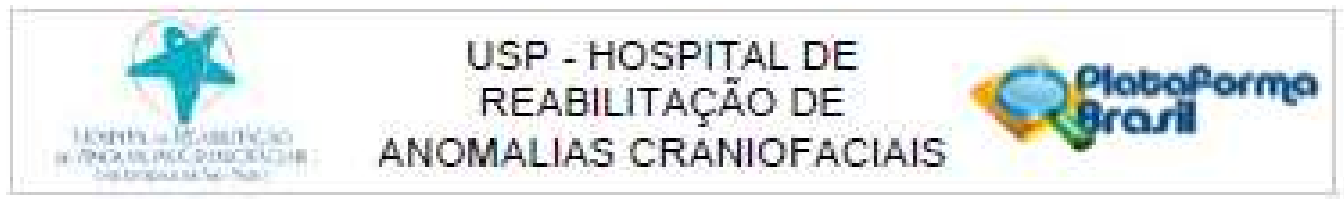

Coranack do Ravear 2 cosese

com otorinolatingologista da equipe. As avallaphles avilologicas foram previamente realzadas no Setor de Fonoaudiologa do HRAClUSP pela fonoaudiologa responsavel da equipe cranlomaxdlotacla. A casuistica incuira padertes ahualmente acompannados pela equipe de cirurgla craniomaxilofadal do hosplta, que ja apresentem em sua documentaç5o cinica o exame de tomograla, e pelo menos uma avaliaç5o audologica. A falxa etaria avalada, incialmente, sera de 6 a 12 anos, porem sera possivel expandir, caso năo se atinja un numero considerado adequado. Pretende-se alcançar uma amcstra de 60 pacientes.

Oojetivo da Pesquisa:

1. Verifcar a presença de matomaģbes auriculares e a presença de perda auditva, classificando-a quanto ao upo e gras;

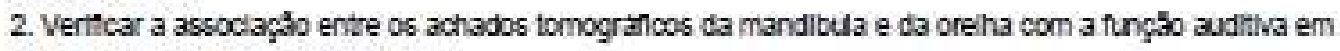
pacientes com dlagnostico de EOAV.

Avallaçso dos Fiscos 6 Beneticios:

Riscos: Nab se aplica.

Beneficios: As pesquisadoras destacam que "os beneficios esperados com o desenvolvimento do presente estudo constluem unra importante contribuliç5o ao conhedimento das condlçbes de

Indviduos com o Espectro Cculoauriculovertebra (EOAV), em relaç̧o a avaliaç5o de aspectos funcionals, especlaimente a acuidade auditiva. Adiclonalmente, o desenvolvimento do projeto podera trazer a populaça-alvo o beneficio verincado em projetos desta natureza".

Comentarios $\theta$ Consideraçses sobre a Pesquilsa:

Os pesquisadores encaminharam emenda com alteraçăo do titulo; exciusăo de um dos objetivos relacionado a analise da mandibuja; e inclusto de trés novos memoros ra equipe de pesquisa. Desta forma, realzaram, acequadamente, todos os ajustes necessarios nos itens e documentos pertinentes ao protocolo de pesquisa.

Consideraç0es sobre os Termos de apresentaça obrigatorla:

Ja apresentados, anteriomente, $\propto 5$ segulntes documentos:

- Carta de encaminhamento dos pesquisadores aos CEP:

- Formulario HRAC.

- Folna de Rosto Platzorma Brasi:

- Termo de Compronisso de Manuselo de informaçles;

- Termo de Pernissbo para uso de Registros para Fins Clentincos:

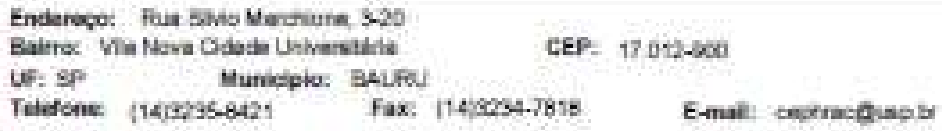




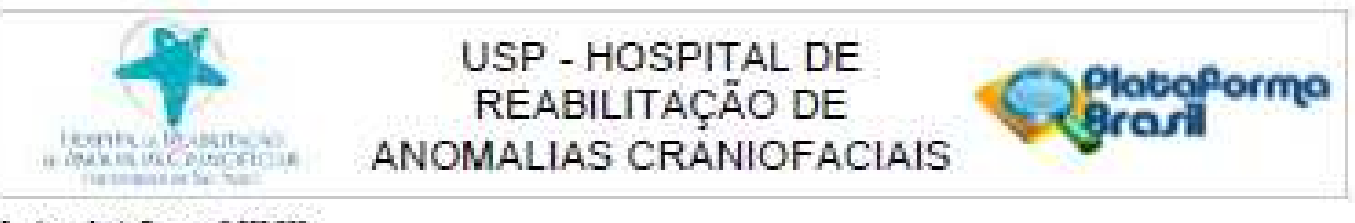

Conthaspe de Pancer zosese

- Termo de Compromisso de Tomar Plbicos os Resultados da Pesquisa e Destinaçăo de Materials ou Dados Coletados:

- Termo de Compronisso do Pesquisador Responsave.

Recomendaçe日:

Nso ha.

Conclusoee ou PendÁnclas $\theta$ Llata de inadequaçes:

Considerando que as atteraples apresentadas nåo ferem a etica, inclico pareser "emenda aprovada".

Consideraçes Finats a criterio do CEP:

O pesquisador deve atentar que o projeto de pesquisa aprovado por este CEP refere-se ao protocolo suometido para avaliaça. Portanto, conforme a Resoluģ50 CNS 466:12, o pesquisador e responsavel por "desenvolver o projeto conforne delineado", se caso houver ateraçles nesse projeto, este CEP devera ser comuricado em emenda via Plataforma Erasli, para nova avalaç̧o.

Cabe ao pesquisador notficar via Piatatorma Brasill o relatorio final para avallaçăo. Os Termos de Consentimento Live e Esclarecidos elou outros Termos corigatorios assinados peios paricipantes da pesqulsa deveráo ser entregues ao CEP. Os relatorios semestrals devem ser nothicacos quando soliciados no parecer.

Este parecer fol eizborado baseado noe documentoes abalxo relacionados:

\begin{tabular}{|c|c|c|c|c|}
\hline Tipo Documento & Arquino & Postagen & ALfíor & Situacso \\
\hline $\begin{array}{l}\text { Iniformaçes B3s c25 } \\
\text { do Proleto }\end{array}$ & 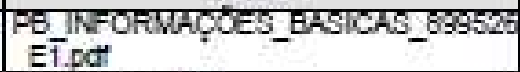 & $\begin{array}{l}07 / 05 / 2017 \\
17: 32: 47\end{array}$ & & AC्Eto \\
\hline Outros & C_Enendapd & $\begin{array}{l}07 / 05 / 2017 \\
17: 23: 06\end{array}$ & Bruna Tozzetti Aves & Acelto \\
\hline Outros & Tem_Comp_Vanuselo_Inform_por & $\begin{array}{l}07 / 05 / 2017 \\
17: 22: 34\end{array}$ & Eruna Tozzetti Aves & Acelto \\
\hline Outros & Fom_Cadzstro_HRAC.pd & $\begin{array}{l}07 / 05 / 2017 \\
17: 21: 45\end{array}$ & Bruna Tozzetti Aves & Acelto \\
\hline $\begin{array}{l}\text { Projeto Detalhado / } \\
\text { Erochura } \\
\text { Investiqador }\end{array}$ & Projeto_Mestuado por & $\begin{array}{l}07 / 05 / 2017 \\
17: 21: 07\end{array}$ & Bruna Tozzettl Avves & Acelto \\
\hline Folha de Rosto & Folha_de_Rosto.pdf & $\begin{array}{l}07 / 05 / 2017 \\
17: 19: 09\end{array}$ & Bruna Tozzettl Aves & Acceito \\
\hline Cutros & Checklst_Prot_Pesq_77_2016.pdf & $\begin{array}{l}10 / 10 / 2016 \\
13: 49: 55\end{array}$ & $\begin{array}{l}\text { Rafael Matios de } \\
\text { Deus }\end{array}$ & Aceito \\
\hline Outros & Termo_de_Compromisso_de_Tornar_ & $05 / 10 / 2016$ & Bruna Tozzetti & Acelto \\
\hline
\end{tabular}

Enderoco: fout SAMs Werchiofe. $\$ 20$

Bairre: Wia Rava Clado Univarstidis

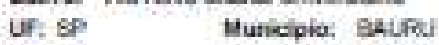

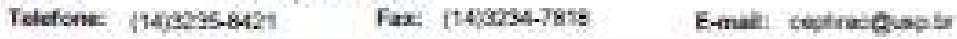




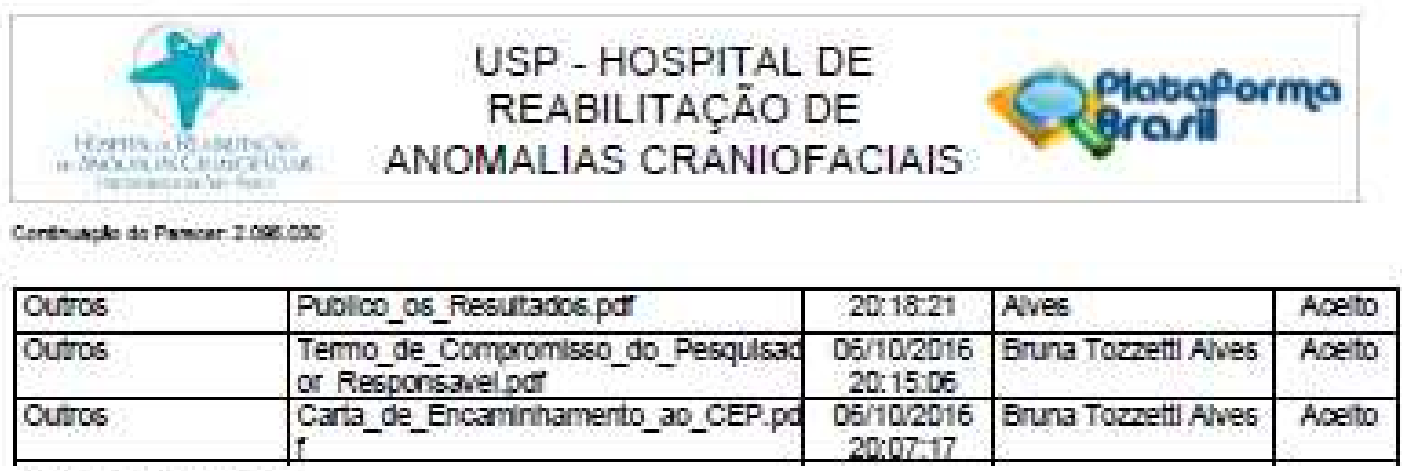

situaça do Parecer:

Aprovado

Necessita Apreclaç3o da CONEP:

Nás

BAURU, Ot de Junho de 2017

Assinado por:

Renata Paclello Yamsehita

(Coordenador) 